\title{
A PDE APPROACH TO FRACTIONAL DIFFUSION: A SPACE-FRACTIONAL WAVE EQUATION*
}

\author{
LEHEL BANJAI ${ }^{\dagger}$ AND ENRIQUE OTÁROLA ${ }^{\ddagger}$
}

\begin{abstract}
We study solution techniques for an evolution equation involving second order derivative in time and the spectral fractional powers, of order $s \in(0,1)$, of symmetric, coercive, linear, elliptic, second-order operators in bounded domains $\Omega$. We realize fractional diffusion as the Dirichlet-to-Neumann map for a nonuniformly elliptic problem posed on the semi-infinite cylinder $\mathcal{C}=\Omega \times(0, \infty)$. We thus rewrite our evolution problem as a quasi-stationary elliptic problem with a dynamic boundary condition and derive space, time, and space-time regularity estimates for its solution. The latter problem exhibits an exponential decay in the extended dimension and thus suggests a truncation that is suitable for numerical approximation. We propose and analyze two fully discrete schemes. The discretization in time is based on finite difference discretization techniques: trapezoidal and leapfrog schemes. The discretization in space relies on the tensorization of a first-degree FEM in $\Omega$ with a suitable $h p$-FEM in the extended variable. For both schemes we derive stability and error estimates.
\end{abstract}

Key words. space-fractional wave equation, fractional diffusion, nonlocal operators, regularity estimates, fully discrete methods, finite elements, stability, error estimates.

AMS subject classifications. 26A33, 35J70, 35R11, 65M12, 65M15. 65M60.

1. Introduction. We are interested in the numerical approximation of an initial boundary value problem for a space-fractional wave equation. Let $\Omega$ be an open and bounded domain in $\mathbb{R}^{n}(n \geq 1)$ with boundary $\partial \Omega$. Given $s \in(0,1)$, a forcing function $f$, and initial data $g$ and $h$, we seek $u$ such that

$$
\left\{\begin{array}{l}
\partial_{t}^{2} u+\mathcal{L}^{s} u=f \text { in } \Omega \times(0, T), \\
u(0)=g \text { in } \Omega, \quad \partial_{t} u(0)=h \text { in } \Omega .
\end{array}\right.
$$

Here, $\mathcal{L}$ denotes the linear, elliptic, self-adjoint, second order, differential operator

$$
\mathcal{L} w=-\operatorname{div}_{x^{\prime}}\left(A \nabla_{x^{\prime}} w\right)+c w,
$$

supplemented with homogeneous Dirichlet boundary conditions. The coefficient $A \in$ $C^{0,1}(\Omega, \mathrm{GL}(n, \mathbb{R}))$ is symmetric and uniformly positive definite and $0 \leq c \in L^{\infty}(\Omega)$. By $\mathcal{L}^{s}$, with $s \in(0,1)$, we denote the spectral fractional powers of the operator $\mathcal{L}$.

One of the most common and studied physical processes is diffusion: the tendency of a substance to evenly spread into an available space. Classical models of diffusion lead to well-known models and even better studied equations. However, in recent times, it has become evident that many of the assumptions that lead to these models are not always satisfactory or even realistic: memory, heterogeneity or a multiscale structure might violate them. In this setting, the assumption of locality does not hold and to describe diffusion one needs to resort to nonlocal operators. Different models of diffusion have been proposed, fractional diffusion being one of them. An incomplete list of problems where fractional diffusion appears includes finance [35, 44,

\footnotetext{
*EO is partially supported by CONICYT through FONDECYT project 3160201.

$\dagger$ Maxwell Institute for Mathematical Sciences, School of Mathematical \& Computer Sciences, Heriot-Watt University, Edinburgh EH14 4AS, UK. 1.banjai@hw.ac.uk.

${ }_{\ddagger}^{\ddagger}$ Departamento de Matemática, Universidad Técnica Federico Santa María, Valparaíso, Chile. enrique.otarola@usm.cl.
} 
13], turbulent flow [14], quasi-geostrophic flows models [11, 31, models of anomalous thermoviscous behaviors [15], peridynamics [19, 48], and image science [25, 24].

The design of efficient solution techniques for problems involving fractional diffusion is intricate, mainly due to the nonlocal character of $\mathcal{L}^{s}$ [7, 8, 9, 12]. Recently, and in order to overcome such a nonlocal feature, it is has been proved useful in numerical analysis the application of the Caffarelli-Silvestre extension [4, 41, 40. When $\mathcal{L}=-\Delta$ and $\Omega=\mathbb{R}^{n}$, i.e., in the case of the Laplacian in the whole space, Caffarelli and Silvestre 9] showed that $\mathcal{L}^{s}$ can be realized as the Dirichlet-to-Neumann map for an extension problem to the upper half-space $\mathbb{R}_{+}^{n+1}$; the extension corresponds to a nonuniformly elliptic PDE. This result was later extended in [8, 12, 49] to bounded domains $\Omega$ and more general operators, thereby obtaining an extension problem posed on the semi-infinite cylinder $\mathcal{C}:=\Omega \times(0, \infty)$. We shall thus rewrite problem (1.1) as the following quasi-stationary elliptic problem with a dynamic boundary condition [3, 17, 18, 52]:

$$
\left\{\begin{array}{l}
-\operatorname{div}\left(y^{\alpha} \mathbf{A} \nabla \mathscr{U}\right)+y^{\alpha} c \mathscr{U}=0 \text { in } \mathcal{C} \times(0, T), \quad \mathscr{U}=0 \text { on } \partial_{L} \mathcal{C} \times(0, T), \\
d_{s} \partial_{t}^{2} \mathscr{U}+\partial_{\nu}^{\alpha} \mathscr{U}=d_{s} f \text { on }(\Omega \times\{0\}) \times(0, T),
\end{array}\right.
$$

with the initial conditions

$$
\mathscr{U}=g \text { on } \Omega \times\{0\}, t=0, \quad \partial_{t} \mathscr{U}=h \text { on } \Omega \times\{0\}, t=0,
$$

where $\partial_{L} \mathcal{C}=\partial \Omega \times[0, \infty)$ corresponds to the lateral boundary of $\mathcal{C}, \alpha=1-2 s \in$ $(-1,1), d_{s}=2^{\alpha} \Gamma(1-s) / \Gamma(s)$ and the conormal exterior derivative of $\mathscr{U}$ at $\Omega \times\{0\}$ is

$$
\partial_{\nu}^{\alpha} \mathscr{U}=-\lim _{y \rightarrow 0^{+}} y^{\alpha} \mathscr{U}_{y}
$$

the limit must be understood in the sense of distributions 9 , 12, 49. We will call $y$ the extended variable and the dimension $n+1$ in $\mathbb{R}_{+}^{n+1}$ the extended dimension of problem (1.2)-(1.3). Finally, $\mathbf{A}=\operatorname{diag}\{A, 1\} \in C^{0,1}(\mathcal{C}, \mathrm{GL}(n+1, \mathbb{R}))$. With the solution $\mathscr{U}$ to the extension problem (1.2)-(1.3) at hand, we can find the solution to (1.1) via [3, 8, 9, 12, 17, 18, 49, 52]:

$$
u=\left.\mathscr{U}\right|_{y=0} .
$$

To the best of the authors knowledge this is the first work that provides a comprehensive treatment of efficient solution techniques for the space-fractional wave equation (1.1). In (1.1), $\mathcal{L}^{s}$ denotes the spectral fractional powers of the operator $\mathcal{L}$. Recently, problem (1.1) has been considered in the literature but with $\mathcal{L}^{s}=(-\Delta)^{s}$ being the integral fractional Laplace operator [34]: the work [2] proposes a discrete scheme that is based on standard Galerkin finite elements for space discretization and the convolution quadrature approach for the discretization in time. We immediately comment that the spectral and integral definitions of the fractional Laplace operator do not coincide. In fact, as shown in 39] their difference is positive and positivity preserving. This, in particular, implies that the boundary behavior of the respective solutions is quite different [10, 28].

The outline of this paper is as follows. In section 2 we introduce some terminology used throughout this work. We recall the definition of the fractional powers of elliptic operators via spectral theory in section 2.1. and in section 2.2 we briefly describe their localization via the Caffarelli-Silvestre extension and introduce the functional 
framework that is suitable for studying problem (1.2). In section 3, we review existence and uniqueness results together with energy-estimates for problems (1.1) and (1.2). In section 4 we present space, time and space-time regularity results for the solution of problem (1.2). The numerical analysis for problem (1.2) begins in section 5 where we introduce a truncated problem on the bounded cylinder $\mathcal{C}_{y}=\Omega \times(0, \mathcal{Y})$ and study some properties of its solution. In section 6 we preset two fully discrete schemes for the truncated version of (1.2) studied in section 5. Both of them are based on the scheme of [4] for space discretization. For time discretization we consider an implicit finite difference discretization scheme and the so-called leapfrog scheme. We derive stability and a priori error estimates for the proposed schemes for all $s \in(0,1)$. In section 7 we comment on some implementation details pertinent to the problem at hand and present numerical experiments in 1D and 2D.

2. Notation and preliminaries. Throughout this work $\Omega$ is an open, bounded, and convex polytopal subset of $\mathbb{R}^{n}(n \geq 1)$ with boundary $\partial \Omega$. We define the semiinfinite cylinder $\mathcal{C}:=\Omega \times(0, \infty)$ and its lateral boundary $\partial_{L} \mathcal{C}:=\partial \Omega \times[0, \infty)$. For $\mathcal{Y}>0$, we define the truncated cylinder with base $\Omega$ and height $\mathcal{Y}$ as $\mathcal{C}_{y}:=\Omega \times(0, \mathcal{Y})$; its lateral boundary is denoted by $\partial_{L} \mathcal{C}_{y}=\partial \Omega \times(0, \mathcal{Y})$. If $x \in \mathbb{R}^{n+1}$, we write $x=\left(x^{\prime}, y\right)$, with $x^{\prime} \in \mathbb{R}^{n}$ and $y \in \mathbb{R}$.

For an open set $D \subset \mathbb{R}^{n}(n \geq 1)$, if $\omega$ is a weight and $p \in(1, \infty)$, we denote the Lebesgue space of $p$-integrable functions with respect to the measure $\omega \mathrm{d} x$ by $L^{p}(\omega, D)$ 29, 32, 51. Similar notation will be used for weighted Sobolev spaces. If $T>0$ and $\phi: D \times(0, T) \rightarrow \mathbb{R}$, we consider $\phi$ as a function of $t$ with values in a Banach space $X, \phi:(0, T) \ni t \mapsto \phi(t) \equiv \phi(\cdot, t) \in X$. For $1 \leq p \leq \infty, L^{p}(0, T ; X)$ is the space of $X$-valued functions whose norm in $X$ is in $L^{p}(0, T)$. This is a Banach space for the norm

$$
\|\phi\|_{L^{p}(0, T ; X)}=\left(\int_{0}^{T}\|\phi(t)\|_{X}^{p} \mathrm{~d} t\right)^{\frac{1}{p}}, \quad 1 \leq p<\infty, \quad\|\phi\|_{L^{\infty}(0, T ; X)}=\operatorname{esssup}_{t \in(0, T)}\|\phi(t)\|_{X} .
$$

Whenever $X$ is a normed space, $X^{\prime}$ denotes its dual and $\|\cdot\|_{X}$ its norm. If, in addition, $Y$ is a normed space, we write $X \hookrightarrow Y$ to indicate continuous embedding. The relation $a \lesssim b$ means $a \leq C b$, with a constant $C$ that neither depends on $a$ or $b$. The value of $C$ might change at each occurrence.

The next result, that follows from Young's inequality for convolutions, will be instrumental in the analysis that we will perform.

Lemma 2.1 (continuity). If $g \in L^{2}(0, T)$ and $\phi \in L^{1}(0, T)$, then the operator

$$
g \mapsto \Phi, \quad \Phi(t)=\phi \star g(t)=\int_{0}^{t} \phi(t-r) g(r) \mathrm{d} r
$$

is continuous from $L^{2}(0, T)$ into itself and $\|\Phi\|_{L^{2}(0, T)} \leq\|\phi\|_{L^{1}(0, T)}\|g\|_{L^{2}(0, T)}$.

Finally, since we assume $\Omega$ to be convex, in what follows we will make use, without explicit mention, of the following regularity result [27]:

$$
\|w\|_{H^{2}(\Omega)} \lesssim\|\mathcal{L} w\|_{L^{2}(\Omega)} \quad \forall w \in H^{2}(\Omega) \cap H_{0}^{1}(\Omega) .
$$

2.1. Fractional powers of second order elliptic operators. We adopt the spectral definition for the fractional powers of the operator $\mathcal{L}$. To define $\mathcal{L}^{s}$, we begin by noticing that $\mathcal{L}$ induces the following inner product $a_{\Omega}(\cdot, \cdot)$ on $H_{0}^{1}(\Omega)$

$$
a_{\Omega}(w, v)=\int_{\Omega}(A \nabla w \cdot \nabla v+c w v) \mathrm{d} x^{\prime}
$$


and that $\mathcal{L}: H_{0}^{1}(\Omega) \ni u \mapsto a_{\Omega}(u, \cdot) \in H^{-1}(\Omega)$ is an isomorphism. The eigenvalue problem:

$$
(\lambda, \varphi) \in \mathbb{R} \times H_{0}^{1}(\Omega) \backslash\{0\}: \quad a_{\Omega}(\varphi, v)=\lambda(\varphi, v)_{L^{2}(\Omega)} \quad \forall v \in H_{0}^{1}(\Omega)
$$

has a countable collection of solutions $\left\{\lambda_{\ell}, \varphi_{\ell}\right\}_{\ell \in \mathbb{N}} \subset \mathbb{R}_{+} \times H_{0}^{1}(\Omega)$ with the real eigenvalues enumerated in increasing order, counting multiplicities, and such that, $\left\{\varphi_{\ell}\right\}_{\ell=1}^{\infty}$ is an orthonormal basis of $L^{2}(\Omega)$ and an orthogonal basis of $\left(H_{0}^{1}(\Omega), a_{\Omega}(\cdot, \cdot)\right)$ [5, 30]. With these eigenpairs at hand, we introduce, for $s \geq 0$, the fractional Sobolev space

$$
\mathbb{H}^{s}(\Omega)=\left\{w=\sum_{\ell=1}^{\infty} w_{\ell} \varphi_{\ell}:\|w\|_{\mathbb{H}^{s}(\Omega)}^{2}:=\sum_{\ell=1}^{\infty} \lambda_{\ell}^{s}\left|w_{\ell}\right|^{2}<\infty\right\},
$$

and denote by $\mathbb{H}^{-s}(\Omega)$ the dual space of $\mathbb{H}^{s}(\Omega)$. The duality pairing between the aforementioned spaces will be denoted by $\langle\cdot, \cdot\rangle$. We notice that, if $s \in\left(0, \frac{1}{2}\right), \mathbb{H}^{s}(\Omega)=$ $H^{s}(\Omega)=H_{0}^{s}(\Omega)$, while, for $s \in\left(\frac{1}{2}, 1\right), \mathbb{H}^{s}(\Omega)$ can be characterized by [36, 37, 50]

$$
\mathbb{H}^{s}(\Omega)=\left\{w \in H^{s}(\Omega): w=0 \text { on } \partial \Omega\right\} .
$$

If $s=\frac{1}{2}$, we have that $\mathbb{H}^{\frac{1}{2}}(\Omega)$ is the so-called Lions-Magenes space $H_{00}^{\frac{1}{2}}(\Omega)$ [36, [50]. If $s \in(1,2]$, owing to (2.1), we have that $\mathbb{H}^{s}(\Omega)=H^{s}(\Omega) \cap H_{0}^{1}(\Omega)$ [23].

The fractional powers of the operator $\mathcal{L}$ are thus defined by

$$
\mathcal{L}^{s}: \mathbb{H}^{s}(\Omega) \rightarrow \mathbb{H}^{-s}(\Omega), \quad \mathcal{L}^{s} w:=\sum_{\ell=1}^{\infty} \lambda_{\ell}^{s} w_{\ell} \varphi_{\ell}, \quad s \in(0,1) .
$$

2.2. Weighted Sobolev spaces. Both extensions, the one by Caffarelli and Silvestre [9] and the ones in [8, 12, 49] for bounded domains $\Omega$ and general elliptic operators, require us to deal with a local but nonuniformly elliptic problem. To provide an analysis for the latter it is thus suitable to define the weighted space

$$
\stackrel{\circ}{H}_{L}^{1}\left(y^{\alpha}, \mathcal{C}\right)=\left\{w \in H^{1}\left(y^{\alpha}, \mathcal{C}\right): w=0 \text { on } \partial_{L} \mathcal{C}\right\} .
$$

Since $\alpha \in(-1,1),|y|^{\alpha}$ belongs to the Muckenhoupt class $A_{2}[20,38$, 51]. The following important consequences thus follow immediately: $H^{1}\left(y^{\alpha}, \mathcal{C}\right)$ is a Hilbert space and $C^{\infty}(\Omega) \cap H^{1}\left(y^{\alpha}, \mathcal{C}\right)$ is dense in $H^{1}\left(|y|^{\alpha}, \mathcal{C}\right)$ (cf. [51, Proposition 2.1.2, Corollary 2.1.6], [33] and [26, Theorem 1]). In addition, as [41, inequality (2.21)] shows, the following weighted Poincaré inequality holds:

$$
\|w\|_{L^{2}\left(y^{\alpha}, \mathcal{C}\right)} \lesssim\|\nabla w\|_{L^{2}\left(y^{\alpha}, \mathcal{C}\right)} \quad \forall w \in \stackrel{\circ}{H}_{L}^{1}\left(y^{\alpha}, \mathcal{C}\right) .
$$

Thus, $\|\nabla w\|_{L^{2}\left(y^{\alpha}, \mathcal{C}\right)}$ is equivalent to the norm in $\stackrel{\circ}{H}_{L}^{1}\left(y^{\alpha}, \mathcal{C}\right)$.

We define the bilinear form $a: \stackrel{\circ}{H}_{L}^{1}\left(y^{\alpha}, \mathcal{C}\right) \times \stackrel{\circ}{H}_{L}^{1}\left(y^{\alpha}, \mathcal{C}\right) \rightarrow \mathbb{R}$ by

$$
a(w, \phi):=\frac{1}{d_{s}} \int_{\mathcal{C}} y^{\alpha}\left(\mathbf{A}(x) \nabla w \cdot \nabla \phi+c\left(x^{\prime}\right) w \phi\right) \mathrm{d} x,
$$

which is continuous and, owing to (2.7), coercive on $\stackrel{\circ}{H}_{L}^{1}\left(y^{\alpha}, \mathcal{C}\right)$. Consequently, it induces an inner product on $\stackrel{\circ}{H}_{L}^{1}\left(y^{\alpha}, \mathcal{C}\right)$ and the following energy norm:

$$
\|w\|_{\mathcal{C}}^{2}:=a(w, w) \sim\|\nabla w\|_{L^{2}\left(y^{\alpha}, \mathcal{C}\right)}^{2} .
$$


For $w \in H^{1}\left(y^{\alpha}, \mathcal{C}\right), \operatorname{tr}_{\Omega} w$ denotes its trace onto $\Omega \times\{0\}$. We recall that, for $\alpha=1-2 s$, 41, Proposition 2.5] yields

$$
\operatorname{tr}_{\Omega} \stackrel{\circ}{L}_{L}^{1}\left(y^{\alpha}, \mathcal{C}\right)=\mathbb{H}^{s}(\Omega), \quad\left\|\operatorname{tr}_{\Omega} w\right\|_{\mathbb{H}^{s}(\Omega)} \leq C_{\mathrm{tr}}\|w\|_{\mathcal{C}}
$$

The seminal work of Caffarelli and Silvestre [9] and its extensions to bounded domains [8, 12, 49] showed that the operator $\mathcal{L}^{s}$ can be realized as the Dirichletto-Neumann map for a nonuniformly elliptic boundary value problem. Namely, if $\mathfrak{U}$ solves

$$
-\operatorname{div}\left(y^{\alpha} \mathbf{A} \nabla \mathfrak{U}\right)+c y^{\alpha} \mathfrak{U}=0 \text { in } \mathcal{C}, \quad \mathfrak{U}=0 \text { on } \partial_{L} \mathcal{C}, \quad \partial_{\nu}^{\alpha} \mathfrak{U}=d_{s} f \text { on } \Omega \times\{0\},
$$

where $\alpha=1-2 s, \partial_{\nu}^{\alpha} \mathfrak{U}=-\lim _{y \downarrow 0} y^{\alpha} \mathfrak{U}_{y}$ and $d_{s}=2^{\alpha} \Gamma(1-s) / \Gamma(s)$ is a normalization constant, then $\mathfrak{u}=\operatorname{tr}_{\Omega} \mathfrak{U} \in \mathbb{H}^{s}(\Omega)$ solves

$$
\mathcal{L}^{s} \mathfrak{u}=f
$$

3. Well-posedness and energy estimates. In this section we briefly review the results of [43] regarding the existence and uniqueness of weak solutions for problems (1.1) and (1.2)-(1.3). We also provide basic energy estimates.

3.1. The fractional wave equation. We assume that the data of problem (1.1) is such that $f \in L^{2}\left(0, T ; L^{2}(\Omega)\right), g \in \mathbb{H}^{s}(\Omega)$ and $h \in L^{2}(\Omega)$ and define

$$
\Lambda(f, g, h):=\|f\|_{L^{2}\left(0, T ; L^{2}(\Omega)\right)}+\|g\|_{\mathbb{H}^{s}(\Omega)}+\|h\|_{L^{2}(\Omega)} .
$$

Definition 3.1 (weak solution for (1.1)). We call $u \in L^{2}\left(0, T ; \mathbb{H}^{s}(\Omega)\right.$ ), with $\partial_{t} u \in L^{2}\left(0, T ; L^{2}(\Omega)\right)$ and $\partial_{t}^{2} u \in L^{2}\left(0, T ; \mathbb{H}^{-s}(\Omega)\right)$, a weak solution of problem (1.1) if $u(0)=g, \partial_{t} u(0)=h$ and a.e. $t \in(0, T)$,

$$
\left\langle\partial_{t}^{2} u, v\right\rangle+\left\langle\mathcal{L}^{s} u, v\right\rangle=\langle f, v\rangle \quad \forall v \in \mathbb{H}^{s}(\Omega),
$$

where $\langle\cdot, \cdot\rangle$ denotes the the duality pairing between $\mathbb{H}^{s}(\Omega)$ and $\mathbb{H}^{-s}(\Omega)$.

The following remark is in order.

REMARK 3.2 (initial conditions). Since a weak solution $u$ of (1.1) satisfies that $u \in L^{2}\left(0, T ; \mathbb{H}^{s}(\Omega)\right), \partial_{t} u \in L^{2}\left(0, T ; L^{2}(\Omega)\right)$ and $\partial_{t}^{2} u \in L^{2}\left(0, T ; \mathbb{H}^{-s}(\Omega)\right)$, an application of [45, Lemma 7.3] reveals that $u \in C\left([0, T] ; L^{2}(\Omega)\right)$ and $\partial_{t} u \in C\left([0, T] ; \mathbb{H}^{-s}(\Omega)\right)$. The initial conditions involved in Definition 3.1 are thus appropriately defined.

Theorem 3.3 (well-posedness). Given $s \in(0,1), f \in L^{2}\left(0, T ; L^{2}(\Omega)\right), g \in$ $\mathbb{H}^{s}(\Omega)$ and $h \in L^{2}(\Omega)$, problem (1.1) has a unique weak solution. In addition,

$$
\|u\|_{L^{\infty}\left(0, T ; \mathbb{H}^{s}(\Omega)\right)}+\left\|\partial_{t} u\right\|_{L^{\infty}\left(0, T ; L^{2}(\Omega)\right)} \lesssim \Lambda(f, g, h),
$$

where the hidden constant is independent of the problem data.

Proof. The desired results can be obtained by slightly modifying the arguments, based on a Galerkin technique, of [22, 36, 45].

3.2. The extended fractional wave equation. We consider the following notion of weak solution for problem (1.2)-(1.3).

Definition 3.4 (extended weak solution). We call $\mathscr{U} \in L^{\infty}\left(0, T ; \stackrel{\circ}{L}_{L}^{1}\left(y^{\alpha}, \mathcal{C}\right)\right)$, with $\operatorname{tr}_{\Omega} \partial_{t} \mathscr{U} \in L^{\infty}\left(0, T ; L^{2}(\Omega)\right)$ and $\operatorname{tr}_{\Omega} \partial_{t}^{2} \mathscr{U} \in L^{2}\left(0, T ; \mathbb{H}^{-s}(\Omega)\right)$, a weak solution of problem (1.2)-(1.3) if $\operatorname{tr}_{\Omega} \mathscr{U}(0)=g, \operatorname{tr}_{\Omega} \partial_{t} \mathscr{U}(0)=h$ and, for a.e. $t \in(0, T)$,

$$
\left\langle\operatorname{tr}_{\Omega} \partial_{t}^{2} \mathscr{U}, \operatorname{tr}_{\Omega} \phi\right\rangle+a(\mathscr{U}, \phi)=\left\langle f, \operatorname{tr}_{\Omega} \phi\right\rangle \quad \forall \phi \in \stackrel{\circ}{H}_{L}^{1}\left(y^{\alpha}, \mathcal{C}\right),
$$


where $\langle\cdot, \cdot\rangle$ denotes the the duality pairing between $\mathbb{H}^{s}(\Omega)$ and $\mathbb{H}^{-s}(\Omega)$ and the bilinear form $a$ is defined as in (2.8).

Remark 3.5 (dynamic boundary condition). Problem (3.3) is an elliptic problem with the following dynamic boundary condition: $\partial_{\nu}^{\alpha} \mathscr{U}=d_{s}\left(f-\operatorname{tr}_{\Omega} \partial_{t}^{2} \mathscr{U}\right)$ on $\Omega \times\{0\}$.

We present the following important localization result [3, 6, 8, 9, 12, 17, 18, 49, 52,

Theorem 3.6 (Caffarelli-Silvestre extension property). Let $s \in(0,1)$. If $f, g$ and $h$ are as in Theorem 3.3, then the unique weak solution of problem (1.1), in the sense of Definition 3.1 satisfies that $u=\operatorname{tr}_{\Omega} \mathscr{U}$, where $\mathscr{U}$ denotes the unique weak solution to problem (1.2) in the sense of Definition 3.4.

We now present the wellposedness of problem (3.3) together with energy estimates for its solution.

Theorem 3.7 (well-posedness). Given $s \in(0,1), f \in L^{2}\left(0, T ; L^{2}(\Omega)\right), g \in$ $\mathbb{H}^{s}(\Omega)$ and $h \in L^{2}(\Omega)$, then problem (1.2)-(1.3) has a unique weak solution in the sense of Definition 3.4. In addition,

$$
\|\nabla \mathscr{U}\|_{L^{\infty}\left(0, T ; L^{2}\left(y^{\alpha}, \mathcal{C}\right)\right)}+\left\|\operatorname{tr}_{\Omega} \partial_{t} \mathscr{U}\right\|_{L^{\infty}\left(0, T ; L^{2}(\Omega)\right)} \lesssim \Lambda(f, g, h),
$$

where the hidden constant is independent of the problem data and $\Lambda(f, g, h)$ is defined as in (3.1).

Proof. See [43, Theorem 3.11].

REMARK 3.8 (initial data). The initial data $g$ and $h$ of problem (3.3) determine $\mathscr{U}(0)$ and $\partial_{t} \mathscr{U}(0)$ only on $\Omega \times\{0\}$ in a trace sense. However, in the analysis that follows it will be necessary to consider their extension to the whole cylinder $\mathcal{C}$. We thus define $\mathscr{U}(0)=\mathcal{E}_{\alpha} g$ and $\mathscr{U}_{t}(0)=\mathcal{E}_{\alpha} h$, where the $\alpha$-harmonic extension operator

$$
\mathcal{E}_{\alpha}: \mathbb{H}^{s}(\Omega) \rightarrow \stackrel{\circ}{H}_{L}^{1}\left(y^{\alpha}, \mathcal{C}\right)
$$

is defined as follows: If $w \in \mathbb{H}^{s}(\Omega)$, then $\mathscr{W}=\mathcal{E}_{\alpha} w \in \stackrel{\circ}{H}_{L}^{1}\left(y^{\alpha}, \mathcal{C}\right)$ solves

$$
\operatorname{div}\left(y^{\alpha} \mathbf{A} \nabla \mathscr{W}\right)+y^{\alpha} c \mathscr{W}=0 \text { in } \mathcal{C}, \quad \mathscr{W}=0 \text { on } \partial_{L} \mathcal{C}, \quad \mathscr{W}=w \text { on } \Omega \times\{0\} .
$$

References [8, 12] provide, for $w \in \mathbb{H}^{s}(\Omega)$, the estimate $\left\|\nabla \mathcal{E}_{\alpha} w\right\|_{L^{2}\left(y^{\alpha}, \mathcal{C}\right)} \lesssim\|w\|_{\mathbb{H}^{s}(\Omega)}$.

3.3. Solution representation. In this section we present a solution representation formula for the solution to problem (3.3). To accomplish this task, we first notice that the solution to problem (1.1) can be written as $u\left(x^{\prime}, t\right)=\sum_{k \in \mathbb{N}} u_{k}(t) \varphi_{k}\left(x^{\prime}\right)$, where, for $k \in \mathbb{N}$, the coefficient $u_{k}(t)$ solves

$$
\partial_{t}^{2} u_{k}(t)+\lambda_{k}^{s} u_{k}(t)=f_{k}(t), \quad t>0, \quad u_{k}(0)=g_{k}, \quad \partial_{t} u_{k}(0)=h_{k},
$$

with $g_{k}=\left(g, \varphi_{k}\right)_{L^{2}(\Omega)}, h_{k}=\left(h, \varphi_{k}\right)_{L^{2}(\Omega)}$, and $f_{k}(t)=\left(f(\cdot, t), \varphi_{k}\right)_{L^{2}(\Omega)}$. We recall that the sequence $\left\{\lambda_{k}, \varphi_{k}\right\}_{k \in \mathbb{N}}$ corresponds to the eigenpairs of the operator $\mathcal{L}$ and are defined by (2.3). Basic computations reveal, for $k \in \mathbb{N}$, that

$$
u_{k}(t)=g_{k} \cos \left(\lambda_{k}^{s / 2} t\right)+h_{k} \lambda_{k}^{-\frac{s}{2}} \sin \left(\lambda_{k}^{s / 2} t\right)+\lambda_{k}^{-\frac{s}{2}} \int_{0}^{t} f_{k}(r) \sin \left(\lambda_{k}^{s / 2}(t-r)\right) \mathrm{d} r .
$$

With these ingredients at hand, we can write the solution $\mathscr{U}$ of problem (3.3) as

$$
\mathscr{U}(x, t)=\sum_{k \in \mathbb{N}} u_{k}(t) \varphi_{k}\left(x^{\prime}\right) \psi_{k}(y)
$$


where, for $\alpha=1-2 s$, the functions $\psi_{k}$ solve

$$
\begin{cases}\frac{\mathrm{d}^{2}}{\mathrm{~d} y^{2}} \psi_{k}(y)+\frac{\alpha}{y} \frac{\mathrm{d}}{\mathrm{d} y} \psi_{k}(y)=\lambda_{k} \psi_{k}(y), & y \in(0, \infty), \\ \psi_{k}(0)=1, & \lim _{y \rightarrow \infty} \psi_{k}(y)=0\end{cases}
$$

If $s=\frac{1}{2}$, we thus have $\psi_{k}(y)=\exp \left(-\sqrt{\lambda_{k}} y\right)$ [8, Lemma 2.10]; more generally, if $s \in(0,1) \backslash\left\{\frac{1}{2}\right\}$, then [12, Proposition 2.1]

$$
\psi_{k}(y)=c_{s}\left(\sqrt{\lambda_{k}} y\right)^{s} K_{s}\left(\sqrt{\lambda_{k}} y\right)
$$

where $c_{s}=2^{1-s} / \Gamma(s)$ and $K_{s}$ denotes the modified Bessel function of the second kind. We refer the reader to [1, Chapter 9.6] and [42, Chapter 7.8] for a comprehensive treatment of the Bessel function $K_{s}$. We immediately comment the following property that the function $\psi_{k}$ satisfies:

$$
\lim _{s \rightarrow \frac{1}{2}} \psi_{k}(y)=\exp \left(-\sqrt{\lambda_{k}} y\right) \quad \forall y>0
$$

In addition, for $a, b \in \mathbb{R}^{+}, a<b$, we have [41, formula (2.33)]

$$
\int_{a}^{b} y^{\alpha}\left(\lambda_{k} \psi_{k}(y)^{2}+\psi_{k}^{\prime}(y)^{2}\right) \mathrm{d} y=\left.y^{\alpha} \psi_{k}(y) \psi_{k}^{\prime}(y)\right|_{a} ^{b}
$$

[41, formula (2.32)]

$$
\left|y^{\alpha} \psi_{k}(y) \psi_{k}^{\prime}(y)\right| \lesssim \lambda_{k}^{s} e^{-\sqrt{\lambda_{k}} y}, \quad y \geq 1,
$$

and [11, formula $(2.31)]$

$$
\lim _{y \downarrow 0^{+}} \frac{y^{\alpha} \psi_{k}^{\prime}(y)}{d_{s} \lambda_{k}^{s}}=-1
$$

4. Regularity. In this section we review and derive space, time, and space-time regularity results for the solution $\mathscr{U}$ of problem (3.3).

4.1. Space regularity. To present the space regularity properties of $\mathscr{U}$, we introduce the weight

$$
\omega_{\beta, \theta}(y)=y^{\beta} e^{\theta y}, \quad 0 \leq \theta<2 \sqrt{\lambda_{1}},
$$

where $\beta \in \mathbb{R}$ will be specified later. With this weight at hand, we define the norm

$$
\|v\|_{L^{2}\left(\omega_{\beta, \theta}, \mathcal{C}\right)}:=\left(\int_{0}^{\infty} \int_{\Omega} \omega_{\beta, \theta}(y)\left|v\left(x^{\prime}, y\right)\right|^{2} \mathrm{~d} x^{\prime} \mathrm{d} y\right)^{\frac{1}{2}} .
$$

We now present the following pointwise, in time, bounds for $\mathscr{U}$ [43, Theorem 4.5].

Proposition 4.1 (pointwise bounds). Let $\mathscr{U}$ solve problem (1.2)-(1.3) for $s \in$ $(0,1)$. Let $0 \leq \sigma<s$ and $0 \leq \nu<1+s$. Then, there exists $\kappa>1$ such that the following estimates hold for all $\ell \in \mathbb{N}_{0}$ :

$$
\begin{aligned}
\left\|\partial_{y}^{\ell+1} \mathscr{U}(\cdot, t)\right\|_{L^{2}\left(\omega_{\alpha+2 \ell-2 \sigma, \theta}, \mathcal{C}\right)}^{2} & \lesssim \kappa^{2(\ell+1)}(\ell+1) !^{2}\|u(\cdot, t)\|_{\mathbb{H}^{\sigma+s}(\Omega)}^{2}, \\
\left\|\nabla_{x^{\prime}} \partial_{y}^{\ell+1} \mathscr{U}(\cdot, t)\right\|_{L^{2}\left(\omega_{\alpha+2(\ell+1)-2 \nu, \theta}, \mathcal{C}\right)}^{2} & \lesssim \kappa^{2(\ell+1)}(\ell+1) !^{2}\|u(\cdot, t)\|_{\mathbb{H}^{\nu+s}(\Omega)}^{2}, \\
\left\|\mathcal{L}_{x^{\prime}} \partial_{y}^{\ell+1} \mathscr{U}(\cdot, t)\right\|_{L^{2}\left(\omega_{\alpha+2(\ell+1)-2 \nu, \theta}, \mathcal{C}\right)}^{2} & \lesssim \kappa^{2(\ell+1)}(\ell+1) !^{2}\|u(\cdot, t)\|_{\mathbb{H}^{1+\nu+s}(\Omega)}^{2} .
\end{aligned}
$$


The hidden constants are independent of $\mathscr{U}, \ell$, and the problem data.

The result below shows the spatial analyticity of $\mathscr{U}$ with respect to the extended variable $y \in(0, \infty)$ : $\mathscr{U}$ belongs to countably normed, power-exponentially weighted Bochner spaces of analytic functions with respect to $y$ taking values in spaces $\mathbb{H}^{r}(\Omega)$.

Proposition 4.2 (space regularity). Let $\mathscr{U}$ solve (1.2)-(1.3) for $s \in(0,1)$. Let $0 \leq \sigma<s$ and $0 \leq \nu<1+s$. Then, there exists $\kappa>1$ such that the following regularity estimates hold for all $\ell \in \mathbb{N}_{0}$ :

$$
\begin{gathered}
\left\|\partial_{y}^{\ell+1} \mathscr{U}\right\|_{L^{2}\left(0, T ; L^{2}\left(\omega_{\alpha+2 \ell-2 \sigma, \theta}, \mathcal{C}\right)\right)}^{2} \lesssim \kappa^{2(\ell+1)}(\ell+1) !^{2}\left(\|g\|_{\mathbb{H}^{\sigma+s}(\Omega)}^{2}+\|h\|_{\mathbb{H}^{\sigma}(\Omega)}^{2}\right. \\
\left.+\|f\|_{L^{2}\left(0, T ; \mathbb{H}^{\sigma}(\Omega)\right)}^{2}\right) \\
\left\|\nabla_{x^{\prime}} \partial_{y}^{\ell+1} \mathscr{U}\right\|_{L^{2}\left(0, T ; L^{2}\left(\omega_{\alpha+2(\ell+1)-2 \nu, \theta}, \mathcal{C}\right)\right)}^{2} \lesssim \kappa^{2(\ell+1)}(\ell+1) !^{2}\left(\|g\|_{\mathbb{H}^{\nu+s}(\Omega)}^{2}+\|h\|_{\mathbb{H}^{\nu}(\Omega)}^{2}\right. \\
\left.+\|f\|_{L^{2}\left(0, T ; \mathbb{H}^{\nu}(\Omega)\right)}^{2}\right)
\end{gathered}
$$

and

$$
\begin{aligned}
\left\|\mathcal{L}_{x^{\prime}} \partial_{y}^{\ell+1} \mathscr{U}\right\|_{L^{2}\left(0, T ; L^{2}\left(\omega_{\alpha+2(\ell+1)-2 \nu, \theta}, \mathcal{C}\right)\right)}^{2} & \kappa^{2(\ell+1)}(\ell+1) !^{2}\left(\|g\|_{\mathbb{H}^{1+\nu+s}(\Omega)}^{2}\right. \\
& \left.+\|h\|_{\mathbb{H}^{1+\nu}(\Omega)}^{2}+\|f\|_{L^{2}\left(0, T ; \mathbb{H}^{1+\nu}(\Omega)\right)}^{2}\right) .
\end{aligned}
$$

The hidden constants are independent of $\mathscr{U}, \ell$, and the problem data.

Proof. In view of (3.8) and the continuity estimate of Lemma 2.1, we conclude, for $k \in \mathbb{N}$, that

$$
\left\|u_{k}\right\|_{L^{2}(0, T)}^{2} \lesssim T g_{k}^{2}+T \lambda_{k}^{-s} h_{k}^{2}+T^{2} \lambda_{k}^{-s}\left\|f_{k}\right\|_{L^{2}(0, T)}^{2} .
$$

The desired estimates (4.6)-(4.8) thus follow directly from (4.3)-(4.5). $\mathrm{Q}$

4.2. Time regularity. We begin this section by defining, for $\ell \in\{1, \ldots, 4\}$,

$$
\Sigma_{\ell}(f, g, h):=\|g\|_{\mathbb{H}^{(\ell+1) s}(\Omega)}+\|h\|_{\mathbb{H}^{\ell e s}(\Omega)}+\|f\|_{L^{2}\left(0, T ; \mathbb{H}^{\ell s}(\Omega)\right)} .
$$

In addition, and to shorten notation, we define

$$
\Xi(f, g, h):=\Sigma_{4}(f, g, h)+\left\|\partial_{t}^{2} f\right\|_{L^{2}\left(0, T ; \mathbb{H}^{s}(\Omega)\right)} .
$$

We now derive regularity estimates in time for the solution $\mathscr{U}$. These estimates will be needed in the analysis of the fully discrete schemes proposed in section 6 .

THEOREM 4.3 (time-regularity). Let $\mathscr{U}$ be the solution to problem (1.2)-(1.3) for $s \in(0,1)$. The following regularity estimates in time hold:

$$
\begin{aligned}
\left\|\partial_{t} \nabla \mathscr{U}\right\|_{L^{\infty}\left(0, T ; L^{2}\left(y^{\alpha}, \mathcal{C}\right)\right)} & \lesssim \Sigma_{1}(f, g, h), \\
\left\|\partial_{t}^{2} \nabla \mathscr{U}\right\|_{L^{\infty}\left(0, T ; L^{2}\left(y^{\alpha}, \mathcal{C}\right)\right)} & \lesssim \Sigma_{2}(f, g, h), \\
\left\|\partial_{t}^{3} \nabla \mathscr{U}\right\|_{L^{\infty}\left(0, T ; L^{2}\left(y^{\alpha}, \mathcal{C}\right)\right)} & \lesssim \Sigma_{3}(f, g, h)+\left\|\partial_{t} f\right\|_{L^{2}\left(0, T ; \mathbb{H}^{s}(\Omega)\right)}, \\
\left\|\partial_{t}^{4} \nabla \mathscr{U}\right\|_{L^{\infty}\left(0, T ; L^{2}\left(y^{\alpha}, \mathcal{C}\right)\right)} & \lesssim \Xi(f, g, h) .
\end{aligned}
$$

In all these inequalities the hidden constants do not depend either on $\mathscr{U}$ or the problem data. 
Proof. Since $\left\{\varphi_{k}\right\}_{k \in \mathbb{N}}$ is an orthonormal basis of $L^{2}(\Omega)$ and an orthogonal basis of $\left(H_{0}^{1}(\Omega), a_{\Omega}(\cdot, \cdot)\right)$, the definition of the energy norm $\|\cdot\|_{\mathcal{C}}$, given in (2.9), and the properties (3.12) and (3.14) allow us to conclude, for $\ell \in \mathbb{N}_{0}$, that

$$
\begin{aligned}
\left\|\partial_{t}^{\ell} \nabla \mathscr{U}(\cdot, t)\right\|_{\mathcal{C}}^{2} & =d_{s}^{-1} \int_{\mathcal{C}} y^{\alpha}\left[\mathbf{A} \nabla \partial_{t}^{\ell} \mathscr{U}(\cdot, t) \cdot \nabla \partial_{t}^{\ell} \mathscr{U}(\cdot, t)+c\left(\partial_{t}^{\ell} \mathscr{U}(\cdot, t)\right)^{2}\right] \mathrm{d} x \\
& =d_{s}^{-1} \sum_{k \in \mathbb{N}}\left(\partial_{t}^{\ell} u_{k}(t)\right)^{2} \int_{0}^{\infty}\left[\lambda_{k} \psi_{k}(y)^{2}+\psi_{k}^{\prime}(y)^{2}\right] \mathrm{d} y=\sum_{k \in \mathbb{N}}\left(\partial_{t}^{\ell} u_{k}(t)\right)^{2} \lambda_{k}^{s} .
\end{aligned}
$$

We have thus arrived at the estimate $\left\|\partial_{t}^{\ell} \nabla \mathscr{U}(\cdot, t)\right\|_{\mathcal{C}}^{2}=\left\|\partial_{t}^{\ell} u(\cdot, t)\right\|_{\mathbb{H}^{s}(\Omega)}^{2}$.

We now invoke the explicit representation of the coefficient $u_{k}(t)$, with $k \in \mathbb{N}$, which is provided in (3.8), to obtain that

$$
\partial_{t} u_{k}(t)=-g_{k} \lambda_{k}^{s / 2} \sin \left(\lambda_{k}^{s / 2} t\right)+h_{k} \cos \left(\lambda_{k}^{s / 2} t\right)+\int_{0}^{t} f_{k}(r) \cos \left(\lambda_{k}^{s / 2}(t-r)\right) \mathrm{d} r
$$

This, on the basis of the definition of the norm $\|\cdot\|_{\mathbb{H}^{r}(\Omega)}$, given in (2.4), and an application of Lemma 2.1, reveal that

$$
\left\|\partial_{t} \nabla \mathscr{U}(\cdot, t)\right\|_{\mathcal{C}}^{2} \lesssim \sum_{k \in \mathbb{N}}\left(\partial_{t} u_{k}(t)\right)^{2} \lambda_{k}^{s} \lesssim\|g\|_{\mathbb{H}^{2 s}(\Omega)}^{2}+\|h\|_{\mathbb{H}^{s}(\Omega)}^{2}+\sum_{k \in \mathbb{N}} \lambda_{k}^{s}\left\|f_{k}\right\|_{L^{2}(0, T)}^{2},
$$

which implies the desired estimate (4.11).

To derive (4.12) we invoke, again, the representation formula (3.8) and write

$$
\begin{aligned}
\partial_{t}^{2} u_{k}(t)=-g_{k} \lambda_{k}^{s} \cos \left(\lambda_{k}^{s / 2} t\right)- & h_{k} \lambda_{k}^{s / 2} \sin \left(\lambda_{k}^{s / 2} t\right) \\
& +f_{k}(t)-\lambda_{k}^{s / 2} \int_{0}^{t} f_{k}(r) \sin \left(\lambda_{k}^{s / 2}(t-r)\right) \mathrm{d} r .
\end{aligned}
$$

We thus use the definition of the norm $\|\cdot\|_{\mathbb{H}^{r}(\Omega)}$ to arrive at (4.12).

The estimates (4.12)-(4.14) follow similar arguments upon taking derivatives to the explicit representation of the coefficient $u_{k}(t)$, with $k \in \mathbb{N}$, provided in (3.8). This concludes the proof.

4.3. Space-time regularity. We present the following regularity result in space and time.

Theorem 4.4 (space-time regularity). Let $\mathscr{U}$ solve (1.2) (1.3) for $s \in(0,1)$. Let $0 \leq \sigma<s$ and $0 \leq \nu<1+s$. Then, there exists $\kappa>1$ such that the following regularity estimates hold for all $\ell \in \mathbb{N}_{0}$ :

$$
\begin{aligned}
& \left\|\partial_{t}^{2} \partial_{y}^{\ell+1} \mathscr{U}\right\|_{L^{2}\left(0, T ; L^{2}\left(\omega_{\alpha+2 \ell-2 \sigma, \theta}, \mathcal{C}\right)\right)}^{2} \lesssim \kappa^{2(\ell+1)}(\ell+1) !^{2}\left(\|g\|_{\mathbb{H}^{\sigma+3 s}(\Omega)}^{2}\right. \\
& \left.+\|h\|_{\mathbb{H}^{\sigma+2 s}(\Omega)}^{2}+\|f\|_{L^{2}\left(0, T ; \mathbb{H}^{\sigma+2 s}(\Omega)\right)}^{2}\right), \\
& \left\|\partial_{t}^{2} \nabla_{x^{\prime}} \partial_{y}^{\ell+1} \mathscr{U}\right\|_{L^{2}\left(0, T ; L^{2}\left(\omega_{\alpha+2(\ell+1)-2 \nu, \theta}, \mathcal{C}\right)\right)}^{2} \lesssim \kappa^{2(\ell+1)}(\ell+1) !^{2}\left(\|g\|_{\mathbb{H}^{\nu+3 s}(\Omega)}^{2}\right. \\
& \left.+\|h\|_{\mathbb{H}^{\nu+2 s}(\Omega)}^{2}+\|f\|_{L^{2}\left(0, T ; \mathbb{H}^{\nu+2 s}(\Omega)\right)}^{2}\right), \\
& \left\|\partial_{t}^{2} \mathcal{L}_{x^{\prime}} \partial_{y}^{\ell+1} \mathscr{U}\right\|_{L^{2}\left(0, T ; L^{2}\left(\omega_{\alpha+2(\ell+1)-2 \nu, \theta}, \mathcal{C}\right)\right)}^{2} \lesssim \kappa^{2(\ell+1)}(\ell+1) !^{2}\left(\|g\|_{\mathbb{H}^{1+\nu+3 s}(\Omega)}^{2}\right. \\
& \left.+\|h\|_{\mathbb{H}^{1+\nu+2 s}(\Omega)}^{2}+\|f\|_{L^{2}\left(0, T ; \mathbb{H}^{1+\nu+2 s}(\Omega)\right)}^{2}\right) .
\end{aligned}
$$


The hidden constants do not depend either on $\mathscr{U}$ or the problem data.

Proof. Similar arguments to the ones used to derive (4.3) reveal that

$$
\left\|\partial_{t}^{2} \partial_{y}^{\ell+1} \mathscr{U}(\cdot, t)\right\|_{L^{2}\left(\omega_{\alpha+2 \ell-2 \sigma, \theta}, \mathcal{C}\right)}^{2} \lesssim \kappa^{2(\ell+1)}(\ell+1) !^{2}\left\|\partial_{t}^{2} u(\cdot, t)\right\|_{\mathbb{H}^{\sigma+s}(\Omega)}^{2} .
$$

To control the right-hand side of the previous inequality we invoke formula (4.15) and the definition of the $\mathbb{H}^{r}(\Omega)$-norm, given in (2.4). These arguments reveal the estimate

$$
\begin{aligned}
\left\|\partial_{t}^{2} u(\cdot, t)\right\|_{\mathbb{H} \sigma+s(\Omega)}^{2} \lesssim & \|g\|_{\mathbb{H} \sigma+3 s(\Omega)}^{2}+\|h\|_{\mathbb{H}^{\sigma+2 s}(\Omega)}^{2} \\
& +\sum_{k \in \mathbb{N}} \lambda_{k}^{\sigma+s}\left(f_{k}^{2}(t)+\lambda_{k}^{s}\left\|f_{k}\right\|_{L^{2}(0, T)}^{2}\right) .
\end{aligned}
$$

This yields (4.16). Similar arguments allow us to derive the regularity estimates (4.17) and (4.18).

As it will be used in the analysis that follows, we introduce

$$
\mathfrak{A}(f, g, h)=\|g\|_{\mathbb{H}^{1+3 s}(\Omega)}+\|h\|_{\mathbb{H}^{1+2 s}(\Omega)}+\|f\|_{L^{2}\left(0, T ; \mathbb{H}^{1+2 s}(\Omega)\right)},
$$

and notice that, if $0 \leq \sigma<s$, then

$$
\begin{aligned}
\left\|\partial_{t}^{2} \partial_{y}^{\ell+1} \mathscr{U}\right\|_{L^{2}\left(0, T ; L^{2}\left(\omega_{\alpha+2 \ell-2 \sigma, \theta}, \mathcal{C}\right)\right)}^{2}+\left\|\partial_{t}^{2} \nabla_{x^{\prime}} \partial_{y}^{\ell+1} \mathscr{U}\right\|_{L^{2}\left(0, T ; L^{2}\left(\omega_{\alpha+2(\ell+1), \theta}, \mathcal{C}\right)\right)}^{2} & \\
+\left\|\partial_{t}^{2} \mathcal{L}_{x^{\prime}} \partial_{y}^{\ell+1} \mathscr{U}\right\|_{L^{2}\left(0, T ; L^{2}\left(\omega_{\alpha+2(\ell+1), \theta}, \mathcal{C}\right)\right)}^{2} & \lesssim \kappa^{2(\ell+1)}(\ell+1) !^{2^{2}} \mathfrak{A}(f, g, h)^{2}
\end{aligned}
$$

As a consequence of the arguments elaborated in the proof of the previous theorem (see (4.19) ) and the previous definition, we can thus immediately arrive at the following regularity estimate.

Corollary 4.5 (space-time regularity). Let u solve (1.1) for $s \in(0,1)$. Then

$$
\left\|\partial_{t}^{2} u\right\|_{\mathbb{H}^{1+s}(\Omega)} \lesssim \mathfrak{A}(f, g, h) .
$$

The hidden constant does not depend either on u or the problem data.

5. Truncation. A first step towards space-discretization is to truncate the semiinfinite cylinder $\mathcal{C}$. In the next result we show that the solution $\mathscr{U}$ to problem (1.2)(1.3) decays exponentially in the extended variable $y$ for a.e. $t \in(0, T)$. This suggests to truncate $\mathcal{C}$ to $\mathcal{C}_{y}=\Omega \times(0, \mathcal{Y})$, with a suitable truncation parameter $\mathcal{Y}$, and seek solutions in this bounded domain.

Proposition 5.1 (exponential decay). Let $s \in(0,1)$ and $\mathscr{U}$ be the solution to (3.3). Then, for every $\mathcal{Y} \geq 1$, we have that

$$
\|\nabla \mathscr{U}\|_{L^{2}\left(0, T ; L^{2}\left(y^{\alpha}, \Omega \times(y, \infty)\right)\right)} \lesssim e^{-\sqrt{\lambda_{1}} \mathscr{Y} / 2} \Lambda(f, g, h),
$$

where $\lambda_{1}$ denotes the first eigenvalue of $\mathcal{L}$ and $\Lambda(f, g, h)$ is defined in (3.1).

Proof. We invoke (3.9) and the fact that $\left\{\varphi_{k}\right\}_{k \in \mathbb{N}}$ is an orthonormal basis of $L^{2}(\Omega)$ and an orthogonal basis of $\left(H_{0}^{1}(\Omega), a_{\Omega}(\cdot, \cdot)\right)$ to conclude that

$$
\int_{0}^{T} \int_{\mathcal{C} \backslash \mathcal{C}_{Y}} y^{\alpha}\left(\mathbf{A} \nabla \mathscr{U} \cdot \nabla \mathscr{U}+c \mathscr{U}^{2}\right) \mathrm{d} x \mathrm{~d} t=\int_{0}^{T} \sum_{k \in \mathbb{N}} u_{k}^{2}(t) \int_{\mathcal{Y}}^{\infty}\left(\lambda_{k} \psi_{k}^{2}+\psi_{k}^{\prime}(y)^{2}\right) \mathrm{d} y \mathrm{~d} t .
$$


We now apply formulas (3.12) and (3.13) to obtain that

$$
\begin{aligned}
\int_{0}^{T} \int_{\mathcal{C} \backslash \mathcal{C}_{y}} y^{\alpha}(\mathbf{A} \nabla \mathscr{U} \cdot \nabla \mathscr{U} & \left.+c \mathscr{U}^{2}\right) \mathrm{d} x \mathrm{~d} t=\sum_{k \in \mathbb{N}}\left|\mathcal{Y}^{\alpha} \psi_{k}(\mathcal{Y}) \psi_{k}^{\prime}(\mathcal{Y})\right| \int_{0}^{T} u_{k}^{2}(t) \mathrm{d} t \\
& \lesssim \sum_{k \in \mathbb{N}} e^{-\sqrt{\lambda_{k}} \mathscr{Y}} \lambda_{k}^{s}\left\|u_{k}\right\|_{L^{2}(0, T)}^{2} \lesssim e^{-\sqrt{\lambda_{1}} \mathcal{Y}}\|u\|_{L^{2}\left(0, T ; \mathbb{H}^{s}(\Omega)\right)}^{2} .
\end{aligned}
$$

The desired estimate (5.1) is thus a consequence of the energy estimate (3.2) for the solution $u$ to problem (1.1).

To describe the truncated version of (3.3), we define the weighted Sobolev space

$$
\stackrel{\circ}{H}_{L}^{1}\left(y^{\alpha}, \mathcal{C}_{y}\right)=\left\{w \in H^{1}\left(y^{\alpha}, \mathcal{C}_{y}\right): w=0 \text { on } \partial_{L} \mathcal{C}_{y} \cup \Omega_{y}\right\},
$$

and the bilinear form $a_{y}: \stackrel{\circ}{H}_{L}^{1}\left(y^{\alpha}, \mathcal{C}_{y}\right) \times \stackrel{\circ}{H}_{L}^{1}\left(y^{\alpha}, \mathcal{C}_{y}\right)$ as

$$
a_{\mathscr{Y}}(w, \phi)=\frac{1}{d_{s}} \int_{\mathcal{C}_{y}} y^{\alpha}\left(\mathbf{A}(x) \nabla w \cdot \nabla \phi+c\left(x^{\prime}\right) w \phi\right) \mathrm{d} x,
$$

where $\mathcal{C}_{y}=\Omega \times(0, \mathcal{Y})$ and $\Omega_{y}=\Omega \times\{\mathscr{Y}\}$.

On the basis of the results of Proposition [5.1, we thus consider the following truncated problem: Find $\mathcal{U} \in L^{\infty}\left(0, T ; \dot{H}_{L}^{1}\left(y^{\alpha}, \mathcal{C}_{y}\right)\right)$ with $\operatorname{tr}_{\Omega} \partial_{t} \mathcal{U} \in L^{\infty}\left(0, T ; L^{2}(\Omega)\right)$ and $\operatorname{tr}_{\Omega} \partial_{t}^{2} \mathcal{U} \in L^{2}\left(0, T ; \mathbb{H}^{-s}(\Omega)\right)$ such that $\operatorname{tr}_{\Omega} \mathcal{U}(0)=g$, $\operatorname{tr}_{\Omega} \partial_{t} \mathcal{U}(0)=h$ and, for a.e. $t \in(0, T)$,

$$
\left\langle\operatorname{tr}_{\Omega} \partial_{t}^{2} \mathcal{U}, \operatorname{tr}_{\Omega} \phi\right\rangle+a_{\mathscr{Y}}(\mathcal{U}, \phi)=\left\langle f, \operatorname{tr}_{\Omega} \phi\right\rangle \quad \forall \phi \in \stackrel{\circ}{H}_{L}^{1}\left(y^{\alpha}, \mathcal{C}_{Y}\right) .
$$

We define $\mathcal{H}_{\alpha}: \mathbb{H}^{s}(\Omega) \rightarrow \stackrel{\circ}{H}_{L}^{1}\left(y^{\alpha}, \mathcal{C}_{y}\right)$, the truncated $\alpha$-harmonic extension operator, as follows: if $w \in \mathbb{H}^{s}(\Omega)$, then $\mathcal{W}=\mathcal{H}_{\alpha} w$ solves

$$
\operatorname{div}\left(y^{\alpha} \mathbf{A} \nabla \mathcal{W}\right)+y^{\alpha} c \mathcal{W}=0 \text { in } \mathcal{C}_{y}, \quad \mathcal{W}=0 \text { on } \partial_{L} \mathcal{C}_{y} \cup \Omega_{y}, \quad \mathcal{W}=w \text { on } \Omega \times\{0\} . \text { (5.4) }
$$

Remark 5.2 (initial data). As in Remark 3.8, we define $\mathcal{U}(0)=\mathcal{H}_{\alpha} g$ and $\mathcal{U}_{t}(0)=\mathcal{H}_{\alpha} h$, where $\mathcal{H}_{\alpha}$ is defined by (5.4). References [8, 12] provide the estimates $\|\mathcal{U}(0)\|_{L^{2}\left(y^{\alpha}, \mathcal{C}\right)} \lesssim\|g\|_{\mathbb{H}^{s}(\Omega)}$ and $\left\|\partial_{t} \mathcal{U}(0)\right\|_{L^{2}\left(y^{\alpha}, \mathcal{C}\right)} \lesssim\|h\|_{\mathbb{H}^{s}(\Omega)}$.

The following result shows that by considering (5.3) instead of (3.3) we only incur an exponentially small error

LEMma 5.3 (exponential error estimate). Let $\mathscr{U}$ and $\mathcal{U}$ be the solutions of problems (3.3) and (5.3), respectively. Then, for every $s \in(0,1)$ and $\mathcal{Y} \geq 1$, we have

$$
\begin{aligned}
\left\|\operatorname{tr}_{\Omega} \partial_{t}(\mathscr{U}-\mathcal{U})\right\|_{L^{\infty}\left(0, T ; L^{2}(\Omega)\right)}^{2}+\|\nabla(\mathscr{U}-\mathcal{U})\|_{L^{\infty}\left(0, T ; L^{2}\left(y^{\alpha}, \mathcal{C}_{\gamma}\right)\right)}^{2} & \lesssim e^{-\sqrt{\lambda_{1}} \gamma / 2} \Sigma_{1}(f, g, h) \\
& \lesssim
\end{aligned}
$$

where $\Sigma_{1}$ is defined by (4.9) and the hidden constant does not depend on either $\mathscr{U}$, $\mathcal{U}$, or the problem data.

Proof. We begin the proof by defining the cutoff function $\rho \in W^{1, \infty}(0, \infty)$ as

$$
\rho(y)=1 \quad 0 \leq y \leq \frac{\mathcal{Y}}{2}, \quad \rho(y)=\frac{2}{\mathscr{Y}}(\mathcal{Y}-y) \quad \frac{\mathcal{Y}}{2}<y<\mathcal{Y}, \quad \rho(y)=0 \quad \mathcal{Y} \geq y .
$$

Notice that by a trivial zero extension we realize that $\mathcal{U}$ belongs to $\stackrel{\circ}{H}_{L}^{1}\left(y^{\alpha}, \mathcal{C}\right)$. We are thus allow to set $\phi=\partial_{t}(\mathcal{U}-\rho \mathscr{U})$ in problems (3.3) and (5.3). With these choices of test functions, we subtract the ensuing equalities and obtain that

$$
\left\langle\operatorname{tr}_{\Omega} \partial_{t}^{2}(\mathcal{U}-\mathscr{U}), \operatorname{tr}_{\Omega} \partial_{t}(\mathcal{U}-\rho \mathscr{U})\right\rangle+a_{\mathscr{Y}}\left(\mathcal{U}-\mathscr{U}, \partial_{t}(\mathcal{U}-\rho \mathscr{U})\right)=0 .
$$


This expression yields

$$
\frac{1}{2} \partial_{t}\left\|\operatorname{tr}_{\Omega} \partial_{t}(\mathcal{U}-\mathscr{U})\right\|_{L^{2}(\Omega)}^{2}+\frac{1}{2} \partial_{t} a_{\mathscr{Y}}(\mathcal{U}-\mathscr{U}, \mathcal{U}-\mathscr{U})=a_{Y}\left(\mathcal{U}-\mathscr{U}, \partial_{t}(\rho \mathscr{U}-\mathscr{U})\right)
$$

We thus integrate over time and use that $\left.\operatorname{tr}_{\Omega} \partial_{t}(\mathcal{U}-\mathscr{U})\right|_{t=0}=0$ to arrive at

$$
\begin{aligned}
& \left\|\operatorname{tr}_{\Omega} \partial_{t}(\mathcal{U}-\mathscr{U})(t)\right\|_{L^{2}(\Omega)}^{2}+\|\nabla(\mathcal{U}-\mathscr{U})(t)\|_{L^{2}\left(y^{\alpha}, \mathcal{C}_{\gamma}\right)}^{2} \\
& \lesssim\|\nabla(\mathcal{U}(0)-\mathscr{U}(0))\|_{L^{2}\left(y^{\alpha}, \mathcal{C}_{\gamma}\right)}^{2}+\int_{0}^{t}\left|a_{\mathscr{Y}}\left(\mathcal{U}-\mathscr{U}, \partial_{\zeta}(\rho \mathscr{U}-\mathscr{U})\right)\right| \mathrm{d} \zeta=: \mathrm{I}+\mathrm{II} .
\end{aligned}
$$

It thus remains to bound the right-hand side of (5.6). First, in view of the fact that $\mathscr{U}(0)=\mathcal{E}_{\alpha} g$ and $\mathcal{U}(0)=\mathcal{H}_{\alpha} g$, with $\mathcal{E}_{\alpha}$ and $\mathcal{H}_{\alpha}$ being defined as in (3.5) and (5.4), respectively, the results of [41, Lemma 3.3] allow us to conclude that

$$
\mathrm{I}=\left\|\nabla\left(\mathcal{H}_{\alpha}-\mathcal{E}_{\alpha}\right) g\right\|_{L^{2}\left(y^{\alpha}, \mathcal{C}_{\gamma}\right)} \lesssim e^{-\sqrt{\lambda}_{1} \gamma / 4}\|g\|_{\mathbb{H}^{s}(\Omega)} .
$$

To bound the term II, we notice that if $y \leq \mathcal{Y} / 2,(\rho-1) \mathscr{U} \equiv 0$. If $y>\mathcal{Y} / 2$, then

$$
\left|\nabla(\rho-1) \partial_{t} \mathscr{U}\right|^{2} \leq 2\left(\frac{4}{\gamma^{2}}\left|\partial_{t} \mathscr{U}\right|^{2}+\left|\partial_{t} \nabla \mathscr{U}\right|^{2}\right) .
$$

Consequently,

$$
\left\|\nabla(\rho-1) \partial_{t} \mathscr{U}\right\|_{L^{2}\left(y^{\alpha}, \mathcal{C}_{y}\right)}^{2} \lesssim \frac{1}{\mathcal{Y}^{2}} \int_{\frac{y}{2}}^{\mathcal{Y}} \int_{\Omega} y^{\alpha}\left|\partial_{t} \mathscr{U}\right|^{2} \mathrm{~d} x^{\prime} \mathrm{d} y+\int_{\frac{y}{2}}^{\mathcal{Y}} \int_{\Omega} y^{\alpha}\left|\partial_{t} \nabla \mathscr{U}\right|^{2} \mathrm{~d} x^{\prime} \mathrm{d} y .
$$

A weighted Poincaré inequality, an application of (3.12) and (3.13), as in the proof of Proposition 5.1. and the use of the estimate (5.1) allow us to conclude that

$$
\begin{aligned}
\left\|\nabla(\rho-1) \partial_{t} \mathscr{U}\right\|_{L^{2}\left(y^{\alpha}, \mathcal{C}_{Y}\right)}^{2} & \lesssim\left\|\partial_{t} \nabla \mathscr{U}\right\|_{L^{2}\left(y^{\alpha}, \Omega \times(y / 2, y)\right)}^{2} \leq\left\|\partial_{t} \nabla \mathscr{U}\right\|_{L^{2}\left(y^{\alpha}, \Omega \times(y / 2, \infty)\right)}^{2} \\
& \lesssim e^{-\sqrt{\lambda_{1}} \gamma / 2}\left\|\partial_{t} \nabla \mathscr{U}\right\|_{L^{2}\left(y^{\alpha}, \mathcal{C}\right)}^{2} .
\end{aligned}
$$

The regularity estimate (4.11) thus implies that

$$
\mathrm{II} \leq C e^{-\sqrt{\lambda}_{1} \gamma / 2} \Sigma_{1}^{2}(f, g, h)+\frac{1}{2}\|\nabla(\mathcal{U}-\mathscr{U})\|_{L^{\infty}\left(0, T ; L^{2}\left(y^{\alpha}, \mathcal{C}_{\gamma}\right)\right)}^{2},
$$

where $C$ denotes a positive constant. Replacing the previous estimate for II and the one in (5.7) for I into (5.6) we obtain the desired exponential error estimate (5.5). $\mathrm{u}$

6. Space and time discretization. In this section we present two fully discrete schemes for approximating the solution to problem (1.1). In view of the localization results of Theorem 3.6 and the exponential error estimate (5.5) we shall thus discretize the truncated problem (5.3). We begin by setting notation on finite element spaces and introducing a finite element approximation in $\Omega$.

6.1. Finite element methods. We follow [4] and present a scheme based on the tensorization of a first-degree FEM in $\Omega$ with a suitable $h p$-FEM in the extended variable. The scheme achieves log-linear complexity with respect to the number of degrees of freedom in $\Omega$. To describe it, on the interval $[0, \mathcal{Y}]$, we consider geometric meshes $\mathcal{G}_{\sigma}^{M}=\left\{I_{m} \mid m=1, \ldots M\right\}$ with $M$ elements and grading factor $\sigma \in(0,1)$ :

$$
I_{1}=\left[0, \mathscr{Y} \sigma^{M-1}\right], \quad I_{i}=\left[\mathscr{Y} \sigma^{M-m+1}, \mathscr{Y} \sigma^{M-m}\right], \quad m \in\{2, \ldots, M\} .
$$


Notice that the meshes $\mathcal{G}_{\sigma}^{M}$ are refined towards $y=0$ in order to capture the singular behavior exhibited by the solution $\mathscr{U}$ on the extended variable $y$ as described in Propositions 4.1 and 4.2. On the aforementioned meshes, we consider a linear degree vector $\boldsymbol{r}=\left(r_{1}, \ldots, r_{M}\right) \in \mathbb{N}^{M}$ with slope $\mathfrak{s}: r_{m}:=\max \{1,\lceil\mathfrak{s} m\rceil\}$, where $m=1,2, \ldots, M$. We define the following finite element space:

$$
S^{\boldsymbol{r}}\left((0, \mathcal{Y}), \mathcal{G}_{\sigma}^{M}\right)=\left\{v_{M} \in C[0, \mathcal{Y}]:\left.v_{M}\right|_{I_{m}} \in \mathbb{P}_{r_{m}}\left(I_{m}\right), I_{m} \in \mathcal{G}_{\sigma}^{M}, m=1, \ldots, M\right\},
$$

and the subspace of $S^{r}\left((0, \mathcal{Y}), \mathcal{G}^{M}\right)$ containing functions that vanish at $y=\mathscr{Y}$ :

$$
S_{\{\mathscr{Y}\}}^{r}\left((0, \mathcal{Y}), \mathcal{G}_{\sigma}^{M}\right)=\left\{v_{M} \in S^{\boldsymbol{r}}\left((0, \mathcal{Y}), \mathcal{G}_{\sigma}^{M}\right): v_{M}(\mathcal{Y})=0\right\} .
$$

Let $\mathscr{T}=\{K\}$ be a conforming partition of $\bar{\Omega}$ into simplices $K$. We denote by $\mathbb{T}$ a collection of conforming and shape regular meshes that are refinements of an original mesh $\mathscr{T}_{0}$. For $\mathscr{T} \in \mathbb{T}$, we define $h_{\mathscr{T}}=\max \{\operatorname{diam}(K): K \in \mathscr{T}\}$ and $N=\# \mathscr{T}$, the number of degrees of freedom of $\mathscr{T}$. We introduce the finite element space:

$$
S_{0}^{1}(\Omega, \mathscr{T})=\left\{v_{h} \in C(\bar{\Omega}):\left.v_{h}\right|_{K} \in \mathbb{P}_{1}(K) \quad \forall K \in \mathscr{T},\left.v_{h}\right|_{\partial \Omega}=0\right\} .
$$

With the meshes $\mathcal{G}_{\sigma}^{M}$ and $\mathscr{T}$ at hand, we define $\mathscr{T}_{\mathcal{Y}}=\mathscr{T} \otimes \mathcal{G}_{\sigma}^{M}$ and the finitedimensional tensor product space

$$
\mathbb{V}_{N, M}^{1, \boldsymbol{r}}\left(\mathscr{T}_{\mathscr{Y}}\right):=S_{0}^{1}(\Omega, \mathscr{T}) \otimes S_{\{\mathscr{r}\}}^{\boldsymbol{r}}\left((0, \mathcal{Y}), \mathcal{G}_{\sigma}^{M}\right) \subset \stackrel{\circ}{H}_{L}^{1}\left(y^{\alpha}, \mathcal{C}_{Y}\right) .
$$

We write $\mathbb{V}\left(\mathscr{T}_{y}\right)$ if the arguments are clear from the context.

Finally, we recall the standard $L^{2}(\Omega)$-orthogonal projection operator $\Pi_{x^{\prime}}$ :

$$
\Pi_{x^{\prime}}: L^{2}(\Omega) \rightarrow S_{0}^{1}(\Omega, \mathscr{T}), \quad\left(\Pi_{x^{\prime}} w, W\right)_{L^{2}(\Omega)}=(w, W)_{L^{2}(\Omega)} \quad \forall W \in S_{0}^{1}(\Omega, \mathscr{T}) .
$$

If $\mathscr{T}$ is quasi-uniform, then [21, Lemma 1.131]

$$
\left\|\Pi_{x^{\prime}} w\right\|_{L^{2}(\Omega)} \leq\|w\|_{L^{2}(\Omega)}, \quad\left\|\nabla \Pi_{x^{\prime}} w\right\|_{L^{2}(\Omega)} \lesssim\|\nabla w\|_{L^{2}(\Omega)} \quad \forall w \in H^{1}(\Omega) .
$$

If, in addition, $w \in H^{2}(\Omega)$, then [21, Proposition 1.134]

$$
\left\|w-\Pi_{x^{\prime}} w\right\|_{L^{2}(\Omega)}+h_{\mathscr{T}}\left\|\nabla\left(w-\Pi_{x^{\prime}} w\right)\right\|_{L^{2}(\Omega)} \lesssim h_{\mathscr{T}}^{2}|v|_{H^{2}(\Omega)} .
$$

6.2. Weighted elliptic projector. We define the weighted elliptic projector

$$
G_{\mathscr{T}_{Y}}: \stackrel{\circ}{H}_{L}^{1}\left(y^{\alpha}, \mathcal{C}_{y}\right) \rightarrow \mathbb{V}\left(\mathscr{T}_{Y}\right)
$$

such that, for $w \in \stackrel{\circ}{H}_{L}^{1}\left(y^{\alpha}, \mathcal{C}_{y}\right)$, it is given by

$$
a_{\mathscr{Y}}\left(G_{\mathscr{T}_{y}} w, W\right)=a_{\mathscr{Y}}(w, W) \quad \forall W \in \mathbb{V}\left(\mathscr{T}_{Y}\right) .
$$

This operator is stable in $\stackrel{\circ}{H}_{L}^{1}\left(y^{\alpha}, \mathcal{C}_{y}\right)$ [40, Proposition 26]:

$$
\left\|\nabla G_{\mathscr{T}_{y}} w\right\|_{L^{2}\left(y^{\alpha}, \mathcal{C}_{Y}\right)} \lesssim\|\nabla w\|_{L^{2}\left(y^{\alpha}, \mathcal{C}_{y}\right)} \quad \forall w \in \stackrel{\circ}{H}_{L}^{1}\left(y^{\alpha}, \mathcal{C}_{Y}\right) .
$$

In what follows we present approximation properties for $G_{\mathscr{T}_{y}}$.

Lemma 6.1 (error estimates for $G_{\mathscr{T}_{\gamma}}$ ). Let $\mathcal{G}_{\sigma}^{M}$ be the geometric mesh defined in (6.1) where $\mathcal{Y} \sim\left|\log h_{\mathscr{T}}\right|$ with a sufficiently large constant. Let $w \in \mathbb{H}^{s}(\Omega)$. If $\mathcal{W}$ 
denotes the truncated $\alpha$-harmonic extension of $w$, then there exists a minimal slope $\mathfrak{s}_{\text {min }}$ such that for linear degree vectors $\boldsymbol{r}$ with slope $\mathfrak{s} \geq \mathfrak{s}_{\text {min }}$ there holds

$$
\left\|\nabla\left(\mathcal{W}-G_{\mathscr{T}_{y}} \mathcal{W}\right)\right\|_{L^{2}\left(y^{\alpha}, \mathcal{C}_{y^{\prime}}\right)} \lesssim h_{\mathscr{T}}\|w\|_{\mathbb{H}^{1+s}(\Omega)} .
$$

In addition, if $\mathscr{W}$ denotes the $\alpha$-harmonic extension of $w$, i.e., the solution to (3.6) with $w \in \mathbb{H}^{s}(\Omega)$ as a datum, then

$$
\left\|\operatorname{tr}_{\Omega}\left(\mathscr{W}-G_{\mathscr{T}_{y}} \mathscr{W}\right)\right\|_{\mathbb{H}^{s}(\Omega)} \lesssim\left\|\nabla\left(\mathscr{W}-G_{\mathscr{T}_{y}} \mathscr{W}\right)\right\|_{L^{2}\left(y^{\alpha}, \mathcal{C}\right)} \lesssim h_{\mathscr{T}}\|w\|_{\mathbb{H}^{1+s}(\Omega)} .
$$

In both inequalities the hidden constants are independent of $\mathcal{W}, \mathscr{W}, w$ and $h_{\mathscr{T}}$.

Proof. Let $\Pi_{y,\{y\}}^{r}$ and $\Pi_{x^{\prime}}$ be the univariate $h p$-interpolation operator of 4 , Section 5.5.1] and the standard $L^{2}(\Omega)$-projection operator defined in (6.3), respectively:

$$
\Pi_{y,\{\mathscr{}}^{r}: C([0, \mathcal{Y}]) \rightarrow S_{\{y\}}^{r}\left((0, \mathcal{Y}), \mathcal{G}_{\sigma}^{M}\right), \quad \Pi_{x^{\prime}}: L^{2}(\Omega) \rightarrow S_{0}^{1}(\Omega, \mathscr{T}) .
$$

Set $W=\Pi_{x^{\prime}} \otimes \Pi_{y,\{y\}}^{r} \mathcal{W}$. Since $W \in \mathbb{V}\left(\mathscr{T}_{y}\right)$, Galerkin orthogonality and definition (6.6) yield

$$
\left\|\nabla\left(\mathcal{W}-G_{\mathscr{T}_{y}} \mathcal{W}\right)\right\|_{L^{2}\left(y^{\alpha}, \mathcal{C}_{\gamma}\right)}^{2} \lesssim a_{\mathscr{Y}}\left(\mathcal{W}-G_{\mathscr{T}_{Y}} \mathcal{W}, \mathcal{W}-W\right) .
$$

It suffices to bound $\|\nabla(\mathcal{W}-W)\|_{L^{2}\left(y^{\alpha}, \mathcal{C}_{y}\right)}$. The stability properties of $\Pi_{x^{\prime}}$, as described in (6.4), reveal that

$$
\|\nabla(\mathcal{W}-W)\|_{L^{2}\left(y^{\alpha}, \mathcal{C}_{\gamma}\right)} \lesssim\left\|\nabla\left(\mathcal{W}-\Pi_{x^{\prime}} \mathcal{W}\right)\right\|_{L^{2}\left(y^{\alpha}, \mathcal{C}_{\gamma}\right)}+\left\|\nabla\left(\mathcal{W}-\Pi_{y,\{\gamma\}}^{r} \mathcal{W}\right)\right\|_{L^{2}\left(y^{\alpha}, \mathcal{C}_{\gamma}\right)}
$$

The estimate (6.8) thus follows from the approximation properties of $\Pi_{x^{\prime}}$ as described in (6.5), the exponential interpolation error estimates of [4, Lemma 5.13], and the regularity properties of $\mathcal{W}$ [4, Theorem 4.7]. The estimate (6.9) follows similar arguments by using first the exponential decay of $\mathscr{W}$ in the extended dimension 41, Proposition 3.1]:

$$
\begin{aligned}
\left\|\nabla\left(\mathscr{W}-G_{\mathscr{T}_{y}} \mathscr{W}\right)\right\|_{L^{2}\left(y^{\alpha}, \mathcal{C}\right)} & \leq\|\nabla \mathscr{W}\|_{L^{2}\left(y^{\alpha}, \mathcal{C} \backslash \mathcal{C}_{\gamma}\right)}+\left\|\nabla\left(\mathscr{W}-G_{\mathscr{T}_{y}} \mathscr{W}\right)\right\|_{L^{2}\left(y^{\alpha}, \mathcal{C}_{\gamma}\right)} \\
& \lesssim e^{-\sqrt{\lambda_{1}} \gamma / 2}+\left\|\nabla\left(\mathscr{W}-G_{\mathscr{T}_{y}} \mathscr{W}\right)\right\|_{L^{2}\left(y^{\alpha}, \mathcal{C}_{\gamma}\right)} .
\end{aligned}
$$

This concludes the proof.

The following improved estimate for the weighted elliptic projection $G_{\mathscr{T}_{y}}$ in the $L^{2}(\Omega)$-norm can be obtained by invoking the estimates of Lemma 6.1 and the arguments elaborated in the proof of [40, Proposition 28].

Lemma $6.2\left(L^{2}(\Omega)\right.$-error estimates for $\left.G_{\mathscr{T}_{\gamma}}\right)$. Let $\mathcal{G}_{\sigma}^{M}$ be the geometric mesh defined in (6.1) where $Y \sim\left|\log h_{\mathscr{T}}\right|$ with a sufficiently large constant. Let $w \in$ $\mathbb{H}^{1+s}(\Omega)$. If $\mathcal{W}$ denotes the truncated $\alpha$-harmonic extension of $w$, then there exists a minimal slope $\mathfrak{s}_{\text {min }}$ such that for linear degree vectors $\boldsymbol{r}$ with slope $\mathfrak{s} \geq \mathfrak{s}_{\text {min }}$ there holds

$$
\left\|\operatorname{tr}_{\Omega}\left(\mathcal{W}-G_{\mathscr{T}_{y}} \mathcal{W}\right)\right\|_{L^{2}(\Omega)} \lesssim h_{\mathscr{T}}^{1+s}\|w\|_{\mathbb{H}^{1+s}(\Omega)} .
$$

In addition, if $\mathscr{W}$ denotes the $\alpha$-harmonic extension of $w$, i.e., the solution to (3.6) with $w \in \mathbb{H}^{1+s}(\Omega)$ as a datum, then

$$
\left\|\operatorname{tr}_{\Omega}\left(\mathscr{W}-G_{\mathscr{T}_{y}} \mathscr{W}\right)\right\|_{L^{2}(\Omega)} \lesssim h_{\mathscr{T}}^{1+s}\|w\|_{\mathbb{H}^{1+s}(\Omega)} .
$$

In both inequalities the hidden constants are independent of $\mathcal{W}, \mathscr{W}, w$ and $h_{\mathscr{T}}$. 
6.3. Time discretization. Let $K \in \mathbb{N}$ be the number of time steps. We define the uniform time step as $\Delta t=T / K$, and we set $t_{k}=k \Delta t, k=0, \ldots, K$. If $\mathcal{X}$ is a normed space with norm $\|\cdot\|_{\mathcal{X}}$, then for $w \in C([0, T] ; \mathcal{X})$ we denote $w_{k}=w\left(t_{k}\right) \in \mathcal{X}$ and $w_{\Delta t}=\left\{w_{k}\right\}_{k=0}^{K} \subset \mathcal{X}$. In addition, for $w_{\Delta t} \subset \mathcal{X}$ and $p \in[1, \infty)$, we define

$$
\left\|w_{\Delta t}\right\|_{\ell^{p}(\mathcal{X})}=\left(\sum_{k=1}^{K} \Delta t\left\|w_{k}\right\|_{\mathcal{X}}^{p}\right)^{\frac{1}{p}}, \quad\left\|w_{\Delta t}\right\|_{\ell^{\infty}(\mathcal{X})}=\max _{0 \leq k \leq K}\left\|w_{k}\right\|_{\mathcal{X}} .
$$

For a sequence of time-discrete functions $w_{\Delta t} \subset \mathcal{X}$, we define, for $k=0, \ldots, K-1$,

$$
\mathfrak{d} w_{k+1}:=(\Delta t)^{-1}\left(w_{k+1}-w_{k}\right), \quad w_{k+1 / 2}:=\frac{1}{2}\left(w_{k+1}+w_{k}\right) .
$$

We also define, for $k=1, \ldots, K-1$,

$$
\mathfrak{c} w_{k}:=\frac{1}{4}\left(w_{k+1}+2 w_{k}+w_{k-1}\right)=\frac{1}{2}\left(w_{k+1 / 2}+w_{k-1 / 2}\right),
$$

and

$$
\mathfrak{d}^{2} w_{k}:=(\Delta t)^{-2}\left(w_{k+1}-2 w_{k}+w_{k-1}\right) .
$$

6.4. Trapezoidal multistep method. Let us now describe our first fully discrete numerical scheme to solve problem (5.3). The space discretization is based on the finite element method on the truncated cylinder $\mathcal{C}_{y}$ described in Section 6.1. The discretization in time is based on a trapezoidal multistep method.

The fully discrete scheme computes the sequence $V_{\Delta t} \subset \mathbb{V}\left(\mathscr{T}_{y}\right)$, an approximation of the solution to (5.3) at each time step. We initialize the scheme by setting

$$
V_{0}=G_{\mathscr{T}_{y}} \mathcal{H}_{\alpha} g, \quad V_{1}=G_{\mathscr{T}_{y}}\left(\mathcal{H}_{\alpha} g+\Delta t \mathcal{H}_{\alpha} h+\frac{1}{2}(\Delta t)^{2} \partial_{t}^{2} \mathcal{U}(0)\right)
$$

where $\mathcal{H}_{\alpha}$ denotes the truncated $\alpha$-harmonic extension and $\partial_{t}^{2} \mathcal{U}(0)=\mathcal{H}_{\alpha} w$ with $w \in \mathbb{H}^{s}(\Omega)$ satisfying

$$
\left\langle w, \operatorname{tr}_{\Omega} \phi\right\rangle=-a_{\mathscr{Y}}(\mathcal{U}(0), \phi)+\left\langle f(0), \operatorname{tr}_{\Omega} \phi\right\rangle \quad \forall \phi \in \stackrel{\circ}{H}_{L}^{1}\left(y^{\alpha}, \mathcal{C}_{y}\right) .
$$

Note that, if $\operatorname{tr}_{\Omega} \phi=0$ the previous equation is satisfied for any $w$.

For $k=1, \ldots, K-1$, let $V_{k+1} \in \mathbb{V}\left(\mathscr{T}_{Y}\right)$ solve

$$
\frac{1}{\Delta t^{2}}\left\langle\operatorname{tr}_{\Omega}\left(V_{k+1}-2 V_{k}+V_{k-1}\right), \operatorname{tr}_{\Omega} W\right\rangle+a_{\mathscr{Y}}\left(\mathfrak{c} V_{k}, W\right)=\left\langle\mathfrak{c} f_{k}, \operatorname{tr}_{\Omega} W\right\rangle
$$

for all $W \in \mathbb{V}\left(\mathscr{T}_{y}\right)$, where $\mathfrak{c} V_{k}$ and $\mathfrak{c} f_{k}$ are defined in (6.15). To obtain an approximate solution to the fractional wave equation (1.1), we define the sequence

$$
U_{\Delta t}=\left\{U_{k}\right\}_{k=0}^{K} \subset S_{0}^{1}(\mathscr{T}, \Omega): \quad U_{\Delta t}:=\operatorname{tr}_{\Omega} V_{\Delta t} .
$$

REMARK 6.3 (locality). The main advantage of problem (6.17)-(6.19) is that it provides an approximated solution to the fractional wave equation (1.1) based on the resolution of the local elliptic problem with a dynamic boundary condition (5.3).

To present the stability of the scheme we introduce, for $k=1, \ldots, K$, the unconditionally nonnegative discrete energy

$$
E_{k}\left(W_{\Delta t}\right):=\frac{1}{2}\left\|\operatorname{tr}_{\Omega} \mathfrak{d} W_{k}\right\|_{L^{2}(\Omega)}^{2}+\frac{1}{2}\left\|W_{k-1 / 2}\right\|_{\mathcal{C}}^{2} .
$$


Lemma 6.4 (energy conservation). If $f \equiv 0$, the fully discrete scheme (6.17) (6.19) conserves energy, i.e., for all $k \in\{1, \ldots, K\}$, we have that

$$
E_{k}\left(V_{\Delta t}\right)=E_{1}\left(V_{\Delta t}\right) \text {. }
$$

If $f \neq 0$, then, for $\ell \in\{1, \ldots, K\}$, we have that

$$
E_{\ell}\left(V_{\Delta t}\right)^{\frac{1}{2}} \leq E_{1}\left(V_{\Delta t}\right)^{\frac{1}{2}}+\frac{1}{\sqrt{2}} \sum_{k=1}^{\ell} \Delta t\left\|\mathfrak{c} f_{k}\right\|_{L^{2}(\Omega)} .
$$

In particular, we have that $E_{K}\left(V_{\Delta t}\right)^{\frac{1}{2}} \leq E_{1}\left(V_{\Delta t}\right)^{\frac{1}{2}}+\frac{1}{\sqrt{2}}\|\mathfrak{c} f\|_{\ell^{1}\left(L^{2}(\Omega)\right)}$.

Proof. Set $W=(2 \Delta t)^{-1}\left(V_{k+1}-V_{k-1}\right)=2^{-1}\left(\mathfrak{d} V_{k+1}+\mathfrak{d} V_{k}\right)=(\Delta t)^{-1}\left(V_{k+1 / 2}-\right.$ $\left.V_{k-1 / 2}\right)$ in (6.19). Basic computations reveal that

$$
\frac{1}{\Delta t}\left(E_{k+1}\left(V_{\Delta t}\right)-E_{k}\left(V_{\Delta t}\right)\right)=\frac{1}{2}\left\langle\mathfrak{c} f_{k}, \operatorname{tr}_{\Omega}\left(\mathfrak{d} V_{k+1}+\mathfrak{d} V_{k}\right)\right\rangle .
$$

If $f \equiv 0$, the previous relation immediately yields (6.22). If $f \neq 0$, an application of the Cauchy-Schwarz inequality allow us to conclude that

$$
E_{k+1}\left(V_{\Delta t}\right)-E_{k}\left(V_{\Delta t}\right) \leq \frac{\Delta t}{\sqrt{2}}\left\|\mathfrak{c} f_{k}\right\|_{L^{2}(\Omega)}\left(E_{k+1}\left(V_{\Delta t}\right)^{\frac{1}{2}}+E_{k}\left(V_{\Delta t}\right)^{\frac{1}{2}}\right)
$$

which yields $E_{k+1}\left(V_{\Delta t}\right)^{\frac{1}{2}}-E_{k}\left(V_{\Delta t}\right)^{\frac{1}{2}} \leq \frac{\Delta t}{\sqrt{2}}\left\|\mathfrak{c} f_{k}\right\|_{L^{2}(\Omega)}$. Adding over $\ell$ we arrive at the desired estimate (6.23). This concludes the proof. $\square$ Let us now show the stability of the scheme.

Lemma 6.5 (stability). The fully discrete scheme (6.17)-(6.19) is stable, namely, for $\ell \in\{1, \ldots, K\}$, we have that

$$
\left\|\operatorname{tr}_{\Omega} \mathfrak{d} V_{\ell}\right\|_{L^{2}(\Omega)}+\left\|V_{\ell-1 / 2}\right\|_{\mathcal{C}} \lesssim\left\|\operatorname{tr}_{\Omega} \mathfrak{d} V_{1}\right\|_{L^{2}(\Omega)}+\left\|V_{1 / 2}\right\|_{\mathcal{C}}+\sum_{k=1}^{\ell} \Delta t\left\|\mathfrak{c} f_{k}\right\|_{L^{2}(\Omega)}
$$

where the hidden constant is independent of $V_{\Delta t}$ and $\Delta t$.

Proof. The proof follows immediately from (6.23).

Let us now present an error analysis for the fully discrete scheme (6.17)-(6.19). To accomplish this task, we introduce the error $e_{\Delta t}:=V_{\Delta t}-\mathcal{U}_{\Delta t}$, and write, as usual

$$
e_{\Delta t}=\left(V_{\Delta t}-G_{\mathscr{T}_{y}} \mathcal{U}_{\Delta t}\right)+\left(G_{\mathscr{T}_{y}} \mathcal{U}_{\Delta t}-\mathcal{U}_{\Delta t}\right)=: \Theta_{\Delta t}+P_{\Delta t} .
$$

The control of $P_{\Delta t}$ follows from (6.8) and (6.11): For $\ell \in\{0,1,2\}$, we have that

$$
\left\|\partial_{t}^{\ell} \nabla P_{\Delta t}\right\|_{\ell^{2}\left(L^{2}\left(y^{\alpha}, \mathcal{C}_{Y}\right)\right)} \lesssim h_{\mathscr{T}} \mathfrak{A}(f, g, h)
$$

and

$$
\left\|\partial_{t}^{\ell} \operatorname{tr}_{\Omega} P_{\Delta t}\right\|_{\ell^{2}\left(L^{2}(\Omega)\right)} \lesssim h_{\mathscr{T}}^{1+s} \mathfrak{A}(f, g, h),
$$

where $\mathfrak{A}(f, g, h)$ is defined in (4.20). Notice that to obtain the estimates (6.26) and (6.27) the regularity estimates of Corollary 4.5 are essential.

In what follows we bound the sequence $\Theta_{\Delta t}$.

Lemma 6.6 (error estimate for $\Theta_{\Delta t}$ ). Let $\mathcal{U}$ be the solution to (5.3) and let $V_{\Delta t}$ be its fully discrete approximation defined as the solution to (6.17)-(6.19). If $\mathfrak{A}(f, g, h)<\infty$ and $\Xi(f, g, h)<\infty$, then

$$
E_{K}\left(\Theta_{\Delta t}\right)^{\frac{1}{2}} \lesssim h_{\mathscr{T}}^{1+s} \mathfrak{A}(f, g, h)+(\Delta t)^{2} \Xi(f, g, h),
$$


where $\Theta_{\Delta t}=V_{\Delta t}-G_{\mathscr{T}_{y}} \mathcal{U}_{\Delta t}, \mathfrak{A}(f, g, h)$ and $\Xi(f, g, h)$ are defined in (4.20) and (4.10), respectively, and the hidden constant is independent of $V_{\Delta t}, \mathcal{U}, \Delta t$, and $h_{\mathscr{T}}$.

Proof. We proceed in three steps.

Step 1. We invoke the continuous problem (5.3), the discrete equation (6.19), and the definition of $G_{\mathscr{T}_{y}}$, given by (6.6), to arrive at the problem that controls the error: For $k=1, \ldots, K-1, \Theta_{k+1} \in \mathbb{V}\left(\mathscr{T}_{Y}\right)$ solves

$$
\begin{aligned}
\frac{1}{\Delta t^{2}}\left\langle\operatorname{tr}_{\Omega}\left(\Theta_{k+1}-2 \Theta_{k}+\Theta_{k-1}\right), \operatorname{tr}_{\Omega} W\right\rangle+a_{\mathscr{Y}}\left(\mathfrak{c} \Theta_{k}, W\right) \\
=\left\langle\operatorname{tr}_{\Omega}\left[\mathfrak{c} \partial_{t}^{2} \mathcal{U}\left(t_{k}\right)-\mathfrak{d}^{2} G_{\mathscr{T}_{y}} \mathcal{U}\left(t_{k}\right)\right], \operatorname{tr}_{\Omega} W\right\rangle \quad \forall W \in \mathbb{V}\left(\mathscr{T}_{y}\right),
\end{aligned}
$$

where $\mathfrak{c} \partial_{t}^{2} \mathcal{U}\left(t_{k}\right)$ and $\mathfrak{d}^{2} G_{\mathscr{T}_{y}} \mathcal{U}\left(t_{k}\right)$ are defined by (6.15) and (6.16), respectively. On the other hand, in view of Remark 5.2 and (6.17), we have that

$$
\Theta_{0}=G_{\mathscr{T}_{\gamma}}\left(\mathcal{H}_{\alpha} g-\mathcal{U}(0)\right)=0, \quad \Theta_{1}=G_{\mathscr{T}_{\gamma}}\left(\mathcal{H}_{\alpha} g+\Delta t \mathcal{H}_{\alpha} h+\frac{1}{2}(\Delta t)^{2} \partial_{t}^{2} \mathcal{U}(0)-\mathcal{U}\left(t_{1}\right)\right)
$$

Now, we write, for $k \geq 1$, the difference $\mathfrak{c} \partial_{t}^{2} \mathcal{U}\left(t_{k}\right)-\mathfrak{d}^{2} G_{\mathscr{T}_{y}} \mathcal{U}\left(t_{k}\right)$ as follows:

$$
\begin{aligned}
\mathfrak{c} \partial_{t}^{2} \mathcal{U}\left(t_{k}\right)-\mathfrak{d}^{2} G_{\mathscr{T}_{y}} \mathcal{U}\left(t_{k}\right)= & {\left[\partial_{t}^{2} \mathcal{U}\left(t_{k}\right)-\mathfrak{d}^{2} \mathcal{U}\left(t_{k}\right)\right]+\left[\mathfrak{d}^{2} \mathcal{U}\left(t_{k}\right)-\mathfrak{d}^{2} G_{\mathscr{T}_{y}} \mathcal{U}\left(t_{k}\right)\right] } \\
& +\frac{\Delta t}{4}\left[\mathfrak{d} \partial_{t}^{2} \mathcal{U}\left(t_{k+1}\right)-\mathfrak{d} \partial_{t}^{2} \mathcal{U}\left(t_{k}\right)\right]=: \mathrm{I}_{k}+\mathrm{II}_{k}+\mathrm{III}_{k} .
\end{aligned}
$$

We thus apply the stability estimate (6.23) to (6.29) and obtain that

$$
E_{K}\left(\Theta_{\Delta t}\right)^{\frac{1}{2}} \leq E_{1}\left(\Theta_{\Delta t}\right)^{\frac{1}{2}}+\frac{1}{\sqrt{2}}\left\|\delta_{\Delta t}\right\|_{\ell^{1}\left(L^{2}(\Omega)\right)},
$$

where $\delta_{\Delta t}=\left\{\delta_{k}\right\}_{k=1}^{K-1}$ and $\delta_{k}=\operatorname{tr}_{\Omega}\left[\mathfrak{c} \partial_{t}^{2} \mathcal{U}\left(t_{k}\right)-\mathfrak{d}^{2} G_{\mathscr{T}_{y}} \mathcal{U}\left(t_{k}\right)\right]$.

Step 2. We proceed to control the term $\left\|\delta_{\Delta t}\right\|_{\ell^{1}\left(L^{2}(\Omega)\right)}$. First, notice that

$$
\left\|\delta_{\Delta t}\right\|_{\ell^{1}\left(L^{2}(\Omega)\right)} \leq \sum_{k=1}^{K-1} \Delta t\left(\left\|\operatorname{tr}_{\Omega} \mathrm{I}_{k}\right\|_{L^{2}(\Omega)}+\left\|\operatorname{tr}_{\Omega} \mathrm{II}_{k}\right\|_{L^{2}(\Omega)}+\left\|\operatorname{tr}_{\Omega} \mathrm{III}_{k}\right\|_{L^{2}(\Omega)}\right) .
$$

To control $\left\|\operatorname{tr}_{\Omega} \mathrm{I}_{k}\right\|_{L^{2}(\Omega)}$ we employ a basic result based on Taylor's Theorem. In fact, for $k \geq 1$, we have that

$$
\left\|\operatorname{tr}_{\Omega} \mathrm{I}_{k}\right\|_{L^{2}(\Omega)} \lesssim(\Delta t)^{2} \sup _{z}\left\|\operatorname{tr}_{\Omega} \partial_{t}^{4} \mathcal{U}(\cdot, z)\right\|_{L^{2}(\Omega)} .
$$

Now, notice that in view of 6.25 we have that $\mathrm{II}_{k}=-\mathfrak{d}^{2} P\left(t_{k}\right)$. The same argument that yields (6.32) allow us to conclude the estimate

$$
\left\|\operatorname{tr}_{\Omega} \mathrm{II}_{k}\right\|_{L^{2}(\Omega)} \lesssim\left\|\operatorname{tr}_{\Omega} \partial_{t}^{2} P\left(t_{k}\right)\right\|_{L^{2}(\Omega)}+(\Delta t)^{2} \sup _{z}\left\|\operatorname{tr}_{\Omega} \partial_{t}^{4} P(\cdot, z)\right\|_{L^{2}(\Omega)} .
$$

We invoke the trace estimate (2.10) and the stability estimate (6.7) of the weighted elliptic projection to conclude, for $z \in(0, T)$, that

$$
\left\|\operatorname{tr}_{\Omega} \partial_{t}^{4}\left(\mathcal{U}-G_{\mathscr{T}_{y}} \mathcal{U}\right)(\cdot, z)\right\|_{L^{2}(\Omega)} \lesssim\left\|\operatorname{tr}_{\Omega} \partial_{t}^{4} \mathcal{U}(\cdot, z)\right\|_{L^{2}(\Omega)}+\left\|\nabla \partial_{t}^{4} \mathcal{U}(\cdot, z)\right\|_{L^{2}\left(y^{\alpha}, \mathcal{C}_{\gamma}\right)} .
$$

Consequently, an application, again, of the trace estimate allows us to conclude that

$$
\left\|\operatorname{tr}_{\Omega} \mathrm{II}_{k}\right\|_{L^{2}(\Omega)} \lesssim h_{\mathscr{T}}^{1+s} \mathfrak{A}(f, g, h)+(\Delta t)^{2}\left\|\nabla \partial_{t}^{4} \mathcal{U}\right\|_{L^{\infty}\left(0, T ; L^{2}\left(y^{\alpha}, \mathcal{C}_{Y}\right)\right.},
$$


where we have used (6.27) with $\ell=2 ; \mathfrak{A}(f, g, h)$ is defined in (4.20).

We finally bound $\mathrm{III}_{k}$. To accomplish this task, we invoke an argument based on Taylor's Theorem. In fact, for $k \geq 1$, we have that

$$
\begin{array}{r}
\left\|\operatorname{tr}_{\Omega} \operatorname{III}_{k}\right\|_{L^{2}(\Omega)}=\frac{\Delta t}{4}\left\|\operatorname{tr}_{\Omega}\left[\partial_{t}^{3} \mathcal{U}\left(\cdot, t_{k+1}\right)+\frac{\Delta t}{2} \partial_{t}^{4} \mathcal{U}(\cdot, \bar{z})-\partial_{t}^{3} \mathcal{U}\left(\cdot, t_{k}\right)-\frac{\Delta t}{2} \partial_{t}^{4} \mathcal{U}(\cdot, \underline{z})\right]\right\|_{L^{2}(\Omega)} \\
\lesssim(\Delta t)^{2}\left\|\operatorname{tr}_{\Omega} \partial_{t}^{4} \mathcal{U}\right\|_{L^{\infty}\left(0, T ; L^{2}(\Omega)\right)}, \quad \text { (6.34) }
\end{array}
$$

where $\underline{z}$ and $\bar{z}$ belong to $\left(t_{k-1}, t_{k+1}\right)$.

Replacing the estimates (6.32), (6.33), and (6.34) into (6.31) we arrive at

$$
\left\|\delta_{\Delta t}\right\|_{\ell^{1}\left(L^{2}(\Omega)\right)} \lesssim h_{\mathscr{T}}^{1+s} \mathfrak{A}(f, g, h)+(\Delta t)^{2}\left\|\partial_{t}^{4} \nabla \mathcal{U}\right\|_{L^{\infty}\left(0, T ; L^{2}\left(y^{\alpha}, \mathcal{C}_{\gamma}\right)\right)} .
$$

Step 3. We bound $E_{1}\left(\Theta_{\Delta t}\right)$. Since $\Theta_{0}=0$, we utilize (6.14) and write

$$
E_{1}\left(\Theta_{\Delta t}\right)=\frac{1}{2}\left\|\operatorname{tr}_{\Omega} \mathfrak{d} \Theta_{1}\right\|_{L^{2}(\Omega)}^{2}+\frac{1}{2}\left\|\Theta_{1 / 2}\right\|_{\mathcal{C}}^{2}=\frac{1}{2(\Delta t)^{2}}\left\|\operatorname{tr}_{\Omega} \Theta_{1}\right\|_{L^{2}(\Omega)}^{2}+\frac{1}{8}\left\|\Theta_{1}\right\|_{\mathcal{C}}^{2} .
$$

In view of the trace estimate (2.10) and the stability property of $G_{\mathscr{T}_{y}}$ given in (6.7) we can thus conclude that

$$
\left\|\operatorname{tr}_{\Omega} \Theta_{1}\right\|_{L^{2}(\Omega)} \lesssim\left\|\Theta_{1}\right\|_{\mathcal{C}} \lesssim\left\|\mathcal{U}(0)+\Delta t \mathcal{U}_{t}(0)+\frac{1}{2}(\Delta t)^{2} \partial_{t}^{2} \mathcal{U}(0)-\mathcal{U}\left(t_{1}\right)\right\|_{\mathcal{C}}
$$

An application of Taylor's Theorem reveals that

$$
\left\|\operatorname{tr}_{\Omega} \Theta_{1}\right\|_{L^{2}(\Omega)} \lesssim\left\|\Theta_{1}\right\|_{\mathcal{C}} \lesssim(\Delta t)^{3}\left\|\partial_{t}^{3} \nabla \mathcal{U}\right\|_{L^{\infty}\left(0, T ; L^{2}\left(y^{\alpha}, \mathcal{C}_{\gamma}\right)\right)},
$$

which immediately yields

$$
E_{1}\left(\Theta_{\Delta t}\right)^{\frac{1}{2}} \lesssim(\Delta t)^{2}\left\|\partial_{t}^{3} \nabla \mathcal{U}\right\|_{L^{\infty}\left(0, T ; L^{2}\left(y^{\alpha}, \mathcal{C}_{y}\right)\right)} .
$$

The desired estimate follows from replacing (6.35) and (6.37) into (6.30) and using the time-regularity results of Theorem $4.3, \mathrm{Q}$

The exponential error estimate of Lemma 5.3 combined with the error estimates of Lemma 6.6 allow us to conclude the following error estimates.

Lemma 6.7 (error estimates for (6.17)-(6.19) ). Let $\mathscr{U}$ be the solution to (3.3) and let $V_{\Delta t}$ be the solution to the fully discrete problem (6.17)-(6.19). If $\mathfrak{A}(f, g, h)<\infty$ and $\Xi(f, g, h)<\infty$, we have the following error estimates:

$$
\left\|\operatorname{tr}_{\Omega}\left(\partial_{t} \mathscr{U}\left(t_{K-1 / 2}\right)-\mathfrak{d} V_{K}\right)\right\|_{L^{2}(\Omega)} \lesssim h_{\mathscr{T}}^{1+s} \mathfrak{A}(f, g, h)+(\Delta t)^{2} \Xi(f, g, h),
$$

and

$$
\left\|\mathscr{U}\left(t_{K-1 / 2}\right)-V_{K-1 / 2}\right\|_{\mathcal{C}} \lesssim h_{\mathscr{T}} \mathfrak{A}(f, g, h)+(\Delta t)^{2} \Xi(f, g, h),
$$

where $\mathfrak{A}(f, g, h)$ and $\Xi(f, g, h)$ are defined by (4.20) and (4.10), respectively, and the hidden constants are independent of $V_{\Delta t}, \mathcal{U}, \mathscr{U}, \Delta t$, and $h_{\mathscr{T}}$.

Proof. We proceed in several steps.

Step 1 . We begin with the following trivial application of the triangle inequality:

$$
\begin{aligned}
\left\|\operatorname{tr}_{\Omega}\left[\partial_{t} \mathscr{U}\left(t_{K-1 / 2}\right)-\mathfrak{d} V_{K}\right]\right\|_{L^{2}(\Omega)} & \leq\left\|\operatorname{tr}_{\Omega} \partial_{t}\left[\mathscr{U}\left(t_{K-1 / 2}\right)-\mathcal{U}\left(t_{K-1 / 2}\right)\right]\right\|_{L^{2}(\Omega)} \\
& +\left\|\operatorname{tr}_{\Omega}\left[\partial_{t} \mathcal{U}\left(t_{K-1 / 2}\right)-\mathfrak{d} V_{K}\right]\right\|_{L^{2}(\Omega)}=: \mathrm{I}+\mathrm{II} .
\end{aligned}
$$


To control the term I we invoke the exponential error estimate (5.5). The latter yields

$$
\mathrm{I}=\left\|\operatorname{tr}_{\Omega} \partial_{t}\left[\mathscr{U}\left(t_{K-1 / 2}\right)-\mathcal{U}\left(t_{K-1 / 2}\right)\right]\right\|_{L^{2}(\Omega)} \lesssim e^{-\sqrt{\lambda_{1}} \gamma / 2} \Sigma_{1}(f, g, h) .
$$

The control of the term II is as follows:

$$
\mathrm{II} \leq\left\|\operatorname{tr}_{\Omega}\left[\partial_{t} \mathcal{U}\left(t_{K-1 / 2}\right)-\mathfrak{d} \mathcal{U}_{K}\right]\right\|_{L^{2}(\Omega)}+\left\|\operatorname{tr}_{\Omega} \mathfrak{d} e_{K}\right\|_{L^{2}(\Omega)}=: \mathrm{II}_{1}+\mathrm{II}_{2},
$$

recalling that $e_{K}=V_{K}-\mathcal{U}_{K}$. Replace the obtained estimates into (6.40). This yields

$$
\left\|\operatorname{tr}_{\Omega}\left[\partial_{t} \mathscr{U}\left(t_{K-1 / 2}\right)-\mathfrak{d} V_{K}\right]\right\|_{L^{2}(\Omega)} \lesssim e^{-\sqrt{\lambda_{1}} \gamma / 2} \Sigma_{1}(f, g, h)+\mathrm{II}_{1}+\mathrm{II}_{2} .
$$

Step 2. The control of $\operatorname{II}_{1}=\left\|\operatorname{tr}_{\Omega}\left[\partial_{t} \mathcal{U}\left(t_{K-1 / 2}\right)-\mathfrak{d} \mathcal{U}_{K}\right]\right\|_{L^{2}(\Omega)}$ follows from a simple application of Taylor's Theorem. In fact, we have that

$$
\mathrm{II}_{1}=\left\|\operatorname{tr}_{\Omega}\left(\partial_{t} \mathcal{U}\left(t_{K-1 / 2}\right)-\frac{\mathcal{U}_{K}-\mathcal{U}_{K-1}}{\Delta t}\right)\right\|_{L^{2}(\Omega)} \lesssim(\Delta t)^{2} \sup _{z}\left\|\operatorname{tr}_{\Omega} \partial_{t}^{3} \mathcal{U}(\cdot, z)\right\|_{L^{2}(\Omega)} .
$$

We now focus on the term $\mathrm{II}_{2}=\left\|\operatorname{tr}_{\Omega} \mathfrak{d} e_{K}\right\|_{L^{2}(\Omega)}$. The triangle inequality yields

$$
\mathrm{II}_{2} \leq\left\|\operatorname{tr}_{\Omega} \mathfrak{d} \Theta_{K}\right\|_{L^{2}(\Omega)}+\left\|\operatorname{tr}_{\Omega} \mathfrak{d} P_{K}\right\|_{L^{2}(\Omega)} .
$$

The results of Lemma 6.6 imply that

$$
\left\|\operatorname{tr}_{\Omega} \mathfrak{d} \Theta_{K}\right\|_{L^{2}(\Omega)} \lesssim h_{\mathscr{T}}^{1+s} \mathfrak{A}(f, g, h)+(\Delta t)^{2} \Xi(f, g, h) .
$$

The control of $\left\|\operatorname{tr}_{\Omega} \mathfrak{d} P_{K}\right\|_{L^{2}(\Omega)}$ follows the same arguments used to bound $\mathrm{II}_{1}$ :

$$
\begin{aligned}
\left\|\operatorname{tr}_{\Omega} \mathfrak{d} P_{K}\right\|_{L^{2}(\Omega)} & =(\Delta t)^{-1}\left\|\operatorname{tr}_{\Omega}\left(P_{K}-P_{K-1}\right)\right\|_{L^{2}(\Omega)} \\
& \lesssim\left\|\operatorname{tr}_{\Omega} \partial_{t} P\left(t_{K-1 / 2}\right)\right\|_{L^{2}(\Omega)}+(\Delta t)^{2}\left\|\operatorname{tr}_{\Omega} \partial_{t}^{3} P\right\|_{L^{\infty}\left(0, T ; L^{2}(\Omega)\right)} .
\end{aligned}
$$

The stability property (6.7) and the estimate (6.27) imply the estimates

$$
\begin{aligned}
\left\|\operatorname{tr}_{\Omega} \mathfrak{d} P_{K}\right\|_{L^{2}(\Omega)} & \lesssim\left\|\operatorname{tr}_{\Omega} \partial_{t} P\right\|_{L^{\infty}\left(0, T ; L^{2}(\Omega)\right)}+(\Delta t)^{2}\left\|\nabla \partial_{t}^{3} \mathcal{U}\right\|_{L^{\infty}\left(0, T ; L^{2}\left(y^{\alpha}, \mathcal{C}_{\gamma}\right)\right.} \\
& \lesssim h_{\mathscr{T}}^{1+s} \mathfrak{A}(f, g, h)+(\Delta t)^{2}\left\|\nabla \partial_{t}^{3} \mathcal{U}\right\|_{L^{\infty}\left(0, T ; L^{2}\left(y^{\alpha}, \mathcal{C}_{\gamma}\right)\right.} .
\end{aligned}
$$

The previous estimate combined with (6.42) allow us to control $\mathrm{II}_{2}$. Replacing the obtained estimate for $\mathrm{II}_{1}$ and $\mathrm{II}_{2}$ into (6.41) yield the desired estimate (6.38).

Step 3. To obtain (6.39) we invoke similar arguments upon using the estimate

$$
\left\|P_{K-1 / 2}\right\|_{\mathcal{C}} \lesssim\left\|P_{K}\right\|_{\mathcal{C}}+\left\|P_{K-1}\right\|_{\mathcal{C}} \lesssim\left\|\nabla P_{\Delta t}\right\|_{\ell^{\infty}\left(L^{2}\left(y^{\alpha}, \mathcal{C}_{\gamma}\right)\right)} \lesssim h_{\mathscr{T}} \mathfrak{A}(f, g, h) .
$$

This concludes the proof.

The following error estimates follow immediately from Lemma 6.7 and show how the fully discrete approximation $U_{\Delta t}$ approximates $u$.

THEOREM 6.8 (error estimates for (6.20)). Let $u$ be the solution to (1.1) and let $U_{\Delta t}$ be its fully discrete approximation defined by (6.20). If $\mathfrak{A}(f, g, h)<\infty$ and $\Xi(f, g, h)<\infty$, then

$$
\left\|\partial_{t} u\left(t_{K-1 / 2}\right)-\mathfrak{d} U_{K}\right\|_{L^{2}(\Omega)} \lesssim h_{\mathscr{T}}^{1+s} \mathfrak{A}(f, g, h)+(\Delta t)^{2} \Xi(f, g, h),
$$

and

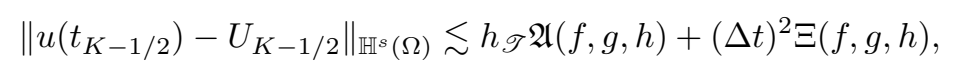

where $\mathfrak{A}(f, g, h)$ and $\Xi(f, g, h)$ are defined by (4.20) and (4.10), respectively, and the hidden constants are independent of $U_{\Delta t}, u, \Delta t$, and $h_{\mathscr{T}}$. 
6.5. The leapfrog scheme. We now present a second fully discrete scheme to approximate the solution to (1.1). To advance in time we use the leapfrog timestepping method while the discretization in space is based on the finite element method described in section 6.1. The scheme computes a sequence $V_{\Delta t} \subset \mathbb{V}\left(\mathscr{T}_{Y}\right)$, an approximation to the solution to (5.3) at each time step. To begin with the description of the scheme, we first initialize it by setting

$$
V_{0}=G_{\mathscr{T}_{\gamma}} \mathcal{H}_{\alpha} g, \quad V_{1}=G_{\mathscr{T}_{y}}\left(\mathcal{H}_{\alpha} g+\Delta t \mathcal{H}_{\alpha} h+\frac{1}{2}(\Delta t)^{2} \partial_{t}^{2} \mathcal{U}(0)\right),
$$

where $\partial_{t}^{2} \mathcal{U}(0)=\mathcal{H}_{\alpha} w$ and $w$ solves (6.18). For $k=1, \ldots, K-1, V_{k+1} \in \stackrel{\circ}{H}_{L}^{1}\left(y^{\alpha}, \mathcal{C}_{y}\right)$ solves

$$
\frac{1}{\Delta t^{2}}\left\langle\operatorname{tr}_{\Omega}\left(V_{k+1}-2 V_{k}+V_{k-1}\right), \operatorname{tr}_{\Omega} W\right\rangle+a_{Y}\left(V_{k}, W\right)=\left\langle f_{k}, \operatorname{tr}_{\Omega} W\right\rangle
$$

for all $W \in \mathbb{V}\left(\mathscr{T}_{y}\right)$. As in the previous section, we define an approximated solution to problem (1.1) as

$$
U_{\Delta t}=\left\{U_{k}\right\}_{k=0}^{K} \subset S_{0}^{1}(\Omega, \mathscr{T}), \quad U_{\Delta t}=\operatorname{tr}_{\Omega} V_{\Delta t} .
$$

In the analysis that follows, the following discrete inverse inequality will be instrumental.

Lemma 6.9 (discrete inverse inequality). Let $\eta \in \mathbb{H}^{-s}(\Omega)$ and let $X \in \mathbb{V}\left(\mathscr{T}_{Y}\right)$ be the solution to

$$
a_{\mathscr{Y}}(X, W)=\left\langle\eta, \operatorname{tr}_{\Omega} W\right\rangle \quad \forall W \in \mathbb{V}\left(\mathscr{T}_{Y}\right) .
$$

We thus have that $\|X\|_{\mathcal{C}} \lesssim\left\|\operatorname{tr}_{\Omega} X\right\|_{\mathbb{H}^{s}(\Omega)}$ and that

$$
\|X\|_{\mathcal{C}} \leq C_{\mathrm{inv}} h_{\mathscr{T}}^{-s}\left\|\operatorname{tr}_{\Omega} X\right\|_{L^{2}(\Omega)}
$$

for some constant $C_{\mathrm{inv}}>0$

Proof. We begin by defining $Z:=\operatorname{tr}_{\Omega} X \in S_{0}^{1}(\mathscr{T}, \Omega)$. There exists $\chi \in \stackrel{\circ}{H}_{L}^{1}\left(y^{\alpha}, \mathcal{C}_{y}\right)$ such that $\operatorname{tr}_{\Omega} \chi=Z$. In fact $\chi=\mathcal{H}_{\alpha} Z$, where $\mathcal{H}_{\alpha}$ is defined in (5.4).

Let us introduce the operator $\tilde{\Pi}=\Pi_{x^{\prime}} \otimes \tilde{\Pi}_{y}^{r}$, where $\tilde{\Pi}_{y}^{r}$ is a slight modification of the operator of [4, Section 5.5.1]: on the first interval $I_{1}$, interpolation at the edge point 0 is used rather than in the middle point of $I_{1}$. The operator $\Pi_{x^{\prime}}$ corresponds to the $L^{2}(\Omega)$-orthogonal projection operator defined in (6.3). Define $\tilde{X}=\tilde{\Pi} \chi$ and notice that the stability properties of $\tilde{\Pi}$ yield

$$
\|\tilde{X}\|_{\mathcal{C}} \lesssim\|\chi\|_{\mathcal{C}}
$$

This, in view of the fact that $\|\chi\|_{\mathcal{C}} \lesssim\|Z\|_{\mathbb{H}^{s}(\Omega)}$, implies $\|\tilde{X}\|_{\mathcal{C}} \lesssim\|Z\|_{\mathbb{H}^{s}(\Omega)}$.

Now, since $Z \in S_{0}^{1}(\mathscr{T}, \Omega)$, we have that $\operatorname{tr}_{\Omega} \tilde{X}=\operatorname{tr}_{\Omega} \chi=Z=\operatorname{tr}_{\Omega} X$ and then that $\operatorname{tr}_{\Omega}(X-\tilde{X})=0$. Since $X-\tilde{X} \in \mathbb{V}\left(\mathscr{T}_{Y}\right)$, we can thus invoke problem (6.48) and conclude that

$$
a_{Y}(X, X-\tilde{X})=\left\langle\eta, \operatorname{tr}_{\Omega}(X-\tilde{X})\right\rangle=0
$$

which yields

$$
\begin{aligned}
a_{\mathscr{Y}}(\tilde{X}, \tilde{X}) & =a_{\mathscr{Y}}((\tilde{X}-X)+X,(\tilde{X}-X)+X) \\
& =a_{\mathscr{Y}}((\tilde{X}-X),(\tilde{X}-X))+a_{\mathscr{Y}}(X, X) \geq a_{\mathscr{Y}}(X, X) .
\end{aligned}
$$


This immediately implies that $\|X\|_{\mathcal{C}} \leq\|\tilde{X}\|_{\mathcal{C}}$, and thus, since $\|\tilde{X}\|_{\mathcal{C}} \lesssim\|Z\|_{\mathbb{H}^{s}(\Omega)}$, that

$$
\|X\|_{\mathcal{C}} \lesssim\|Z\|_{\mathbb{H}^{s}(\Omega)}
$$

Since $Z=\operatorname{tr}_{\Omega} X$, we have thus obtained the desired estimate $\|X\|_{\mathcal{C}} \lesssim\left\|\operatorname{tr}_{\Omega} X\right\|_{\mathbb{H}^{s}(\Omega)}$. The estimate (6.49) thus follows from, for instance, the results of [16].

To analyze the fully discrete scheme (6.45) - (6.46), we define, for $k=1, \ldots, K$, the discrete energy

$$
\mathscr{E}_{k}\left(W_{\Delta t}\right):=\frac{1}{2}\left\|\operatorname{tr}_{\Omega} \mathfrak{d} W_{k}\right\|_{L^{2}(\Omega)}^{2}+\frac{1}{2} a_{y}\left(W_{k}, W_{k-1}\right),
$$

where the bilinear form $a_{y}$ is defined in (5.2).

In the result that follows we show the nonnegativity of the discrete energy $\mathscr{E}_{k}$ under the following CFL condition: $\Delta t$ is chosen to be sufficiently small such that

$$
1-C_{\mathrm{inv}}^{2} \frac{(\Delta t)^{2}}{2 h_{\mathscr{T}}^{2 s}} \geq \theta>0, \quad \theta \in(0,1)
$$

The constant $C_{\text {inv }}$ is as in (6.49).

Lemma 6.10 (CFL condition and nonnegativity of $\mathscr{E}_{k}$ ). If (6.51) holds, then

$$
\mathscr{E}_{k}\left(V_{\Delta t}\right) \geq \frac{\theta}{2}\left\|\operatorname{tr}_{\Omega} \mathfrak{d} V_{k}\right\|_{L^{2}(\Omega)}^{2}+\frac{1}{4}\left[\left\|V_{k}\right\|_{\mathcal{C}}^{2}+\left\|V_{k-1}\right\|_{\mathcal{C}}^{2}\right] \geq 0
$$

for all $k \in\{1, \cdots, K\}$.

Proof. We invoke the inverse inequality (6.49) and thus the CFL condition (6.51) to arrive at

$$
\begin{aligned}
2 a_{\mathscr{Y}}\left(V_{k}, V_{k-1}\right) & =\left\|V_{k}\right\|_{\mathcal{C}}^{2}+\left\|V_{k-1}\right\|_{\mathcal{C}}^{2}-\left\|V_{k}-V_{k-1}\right\|_{\mathcal{C}}^{2} \\
& \geq\left\|V_{k}\right\|_{\mathcal{C}}^{2}+\left\|V_{k-1}\right\|_{\mathcal{C}}^{2}-C_{\mathrm{inv}}^{2} h_{\mathscr{T}}^{-2 s}\left\|\operatorname{tr}_{\Omega}\left(V_{k}-V_{k-1}\right)\right\|_{L^{2}(\Omega)}^{2} \\
& \geq\left\|V_{k}\right\|_{\mathcal{C}}^{2}+\left\|V_{k-1}\right\|_{\mathcal{C}}^{2}+2(\theta-1)\left\|\operatorname{tr}_{\Omega} \mathfrak{d} V_{k}\right\|_{L^{2}(\Omega)}^{2},
\end{aligned}
$$

where, in the last step, we have used definition (6.14). Consequently,

$$
\mathscr{E}_{k}\left(V_{\Delta t}\right) \geq \frac{1}{2}\left\|\operatorname{tr}_{\Omega} \mathfrak{d} V_{k}\right\|_{L^{2}(\Omega)}^{2}+\frac{1}{4}\left\|V_{k}\right\|_{\mathcal{C}}^{2}+\frac{1}{4}\left\|V_{k-1}\right\|_{\mathcal{C}}^{2}+\frac{(\theta-1)}{2}\left\|\operatorname{tr}_{\Omega} \mathfrak{d} V_{k}\right\|_{L^{2}(\Omega)}^{2},
$$

which immediately yields (6.52). This concludes the proof. $\mathrm{Q}$

Lemma 6.11 (energy conservation). If $f \equiv 0$, the fully discrete scheme (6.45)(6.46) conserves energy, i.e., for all $k \in\{1, \ldots, K\}$, we have that

$$
\mathscr{E}_{k}\left(V_{\Delta t}\right)=\mathscr{E}_{1}\left(V_{\Delta t}\right)
$$

If $f \neq 0$, then, for $\ell \in\{1, \ldots, K\}$, we have that

$$
\mathscr{E}_{\ell}\left(V_{\Delta t}\right)^{\frac{1}{2}} \leq \mathscr{E}_{1}\left(V_{\Delta t}\right)^{\frac{1}{2}}+\frac{1}{\sqrt{2 \theta}} \sum_{k=1}^{\ell} \Delta t\left\|f_{k}\right\|_{L^{2}(\Omega)} .
$$

In particular, we have that $\mathscr{E}_{K}\left(V_{\Delta t}\right)^{\frac{1}{2}} \leq \mathscr{E}_{1}\left(V_{\Delta t}\right)^{\frac{1}{2}}+\frac{1}{\sqrt{2 \theta}}\|f\|_{\ell^{1}\left(L^{2}(\Omega)\right)}$.

Proof. Set $W=(2 \Delta t)^{-1}\left(V_{k+1}-V_{k-1}\right)=2^{-1}\left(\mathfrak{d} V_{k+1}+\mathfrak{d} V_{k}\right)$ in (6.46). This yields

$$
\frac{1}{\Delta t}\left(\mathscr{E}_{k+1}\left(V_{\Delta t}\right)-\mathscr{E}_{k}\left(V_{\Delta t}\right)\right)=\frac{1}{2}\left\langle f_{k}, \operatorname{tr}_{\Omega}\left(\mathfrak{d} V_{k+1}+\mathfrak{d} V_{k}\right)\right\rangle
$$


In the case that $f \equiv 0,(6.55)$ immediately yields (6.53). If $f \neq 0$, a trivial application of the Cauchy-Schwarz inequality reveals that

$$
\mathscr{E}_{k+1}\left(V_{\Delta t}\right)-\mathscr{E}_{k}\left(V_{\Delta t}\right) \leq \frac{\Delta t}{2}\left\|f_{k}\right\|_{L^{2}(\Omega)}\left(\left\|\operatorname{tr}_{\Omega} \mathfrak{d} V_{k+1}\right\|_{L^{2}(\Omega)}+\left\|\mathfrak{d} V_{k}\right\|_{L^{2}(\Omega)}\right) .
$$

Invoke the estimate (6.52) and conclude, for $k \in\{1, \ldots, K-1\}$, that $\mathscr{E}_{k}\left(V_{\Delta t}\right) \geq$ $(\theta / 2)\left\|\operatorname{tr}_{\Omega} \mathfrak{d} V_{k}\right\|_{L^{2}(\Omega)}^{2}$. Thus,

$$
\mathscr{E}_{k+1}\left(V_{\Delta t}\right)-\mathscr{E}_{k}\left(V_{\Delta t}\right) \leq \frac{\Delta t}{\sqrt{2 \theta}}\left\|f_{k}\right\|_{L^{2}(\Omega)}\left(\mathscr{E}_{k+1}^{\frac{1}{2}}\left(V_{\Delta t}\right)+\mathscr{E}_{k}^{\frac{1}{2}}\left(V_{\Delta t}\right)\right)
$$

Consequently, we arrive at $\mathscr{E}_{k+1}\left(V_{\Delta t}\right)^{\frac{1}{2}}-\mathscr{E}_{k}\left(V_{\Delta t}\right)^{\frac{1}{2}} \leq(\Delta t / \sqrt{2 \theta})\left\|f_{k}\right\|_{L^{2}(\Omega)}$ which, by adding over $\ell$, yields (6.54). This concludes the proof. $\square$

Lemma 6.12 (stability). The fully discrete scheme (6.45) 6.45 is stable: for $\ell \in\{1, \ldots, K\}$, we have that

$$
\left\|\operatorname{tr}_{\Omega} \mathfrak{d} V_{\ell}\right\|_{L^{2}(\Omega)}+\left\|V_{\ell}\right\|_{\mathcal{C}} \lesssim\left\|\operatorname{tr}_{\Omega} \mathfrak{d} V_{1}\right\|_{L^{2}(\Omega)}+\left\|V_{0}\right\|_{\mathcal{C}}+\left\|V_{1}\right\|_{\mathcal{C}}+\sum_{k=1}^{\ell} \Delta t\left\|f_{k}\right\|_{L^{2}(\Omega)}
$$

where the hidden constant is independent of $V_{\Delta t}, \Delta t$ and $h_{\mathscr{T}}$ but depends on $\theta$.

Proof. We begin by noticing that, in view of (6.52), we arrive at

$$
\left\|\operatorname{tr}_{\Omega} \mathfrak{d} V_{\ell}\right\|_{L^{2}(\Omega)}+\left\|V_{\ell}\right\|_{\mathcal{C}} \lesssim\left(\theta^{-1 / 2}+1\right) \mathscr{E}_{l}\left(V_{\Delta t}\right)^{\frac{1}{2}}
$$

Now, since

$2 a_{\mathscr{Y}}\left(V_{1}, V_{0}\right)=a_{\mathscr{Y}}\left(V_{1}, V_{1}\right)+a_{\mathscr{Y}}\left(V_{0}, V_{0}\right)-a_{\mathscr{Y}}\left(V_{1}-V_{0}, V_{1}-V_{0}\right) \leq a_{\mathscr{Y}}\left(V_{1}, V_{1}\right)+a_{\mathscr{Y}}\left(V_{0}, V_{0}\right)$, an application of the estimate (6.54) allows us to conclude that

$$
\mathscr{E}_{\ell}\left(V_{\Delta t}\right)^{\frac{1}{2}} \lesssim\left\|\operatorname{tr}_{\Omega} \mathfrak{d} V_{1}\right\|_{L^{2}(\Omega)}+\left\|V_{0}\right\|_{\mathcal{C}}+\left\|V_{1}\right\|_{\mathcal{C}}+\frac{1}{\sqrt{\theta}} \sum_{k=1}^{\ell} \Delta t\left\|f_{k}\right\|_{L^{2}(\Omega)} .
$$

The desired estimate (6.56) thus follows from replacing the previous estimate into (6.57). This concludes the proof. $\square$

We now present error estimates for the fully discrete approximation $U_{\Delta t}$ defined in (6.47) that is based on the solution $V_{\Delta t}$ to the fully discrete scheme (6.45)-(6.46). The arguments are similar to the ones used to prove the results in Lemma 6.6. Lemma 6.7, and Theorem 6.8, For brevity we leave details to the reader.

THEOREM 6.13 (error estimates for (6.47)). Let $u$ be the solution to (1.1) and let $U_{\Delta t}$ be its fully discrete approximation defined by (6.47). If $\mathfrak{A}(f, g, h)<\infty$ and $\Xi(f, g, h)<\infty$, then

$$
\left\|\partial_{t} u\left(t_{K-1 / 2}\right)-\mathfrak{d} U_{K}\right\|_{L^{2}(\Omega)} \lesssim h_{\mathscr{T}}^{1+s} \mathfrak{A}(f, g, h)+(\Delta t)^{2} \Xi(f, g, h)
$$

and

$$
\left\|u\left(t_{K}\right)-U_{K}\right\|_{\mathbb{H}^{s}(\Omega)} \lesssim h_{\mathscr{T}} \mathfrak{A}(f, g, h)+(\Delta t)^{2} \Xi(f, g, h),
$$

where $\mathfrak{A}(f, g, h)$ and $\Xi(f, g, h)$ are defined by (4.20) and (4.10), respectively, and the hidden constants are independent of $U_{\Delta t}, u, \Delta t$, and $h_{\mathscr{T}}$. 
6.6. Computable data. In (6.17) we considered

$$
V_{0}=G_{\mathscr{T}_{\gamma}} \mathcal{H}_{\alpha} g, \quad V_{1}=G_{\mathscr{T}_{\gamma}}\left(\mathcal{H}_{\alpha} g+\Delta t \mathcal{H}_{\alpha} h+\frac{1}{2}(\Delta t)^{2} \partial_{t}^{2} \mathcal{U}(0)\right),
$$

as initial data for the fully discrete schemes of sections 6.4 and 6.5. Since the action of $\mathcal{H}_{\alpha}$ involves the resolution of a problem posed on an infinite dimensional space, we immediately conclude that the initial data $V_{0}$ and $V_{1}$ are not computable.

To overcome this deficiency, we introduce the discrete extension operator $\mathcal{H}_{\alpha}^{\mathscr{T}}$, which is defined as follows: if $e \in S_{0}^{1}(\Omega, \mathscr{T})$, then

$$
\mathcal{H}_{\alpha}^{\mathscr{T}} e:=E \in \mathbb{V}\left(\mathscr{T}_{Y}\right): \quad a_{\mathscr{Y}}(E, W)=0 \quad \forall W \in \mathbb{V}\left(\mathscr{T}_{Y}\right): \operatorname{tr}_{\Omega} W=0, \quad \operatorname{tr}_{\Omega} E=e .
$$

With $\mathcal{H}_{\alpha}^{\mathscr{T}}$ at hand, we define the following computable initial data:

$$
\widetilde{V}_{0}=\mathcal{H}_{\alpha}^{\mathscr{T}} \Pi_{x^{\prime}} g, \quad \widetilde{V}_{1}=\mathcal{H}_{\alpha}^{\mathscr{T}}\left(\Pi_{x^{\prime}} g+\Delta t \Pi_{x^{\prime}} h+\frac{1}{2}(\Delta t)^{2} Z\right) .
$$

$Z \in S_{0}^{1}(\mathscr{T}, \Omega)$ solves $\left\langle Z, \operatorname{tr}_{\Omega} W\right\rangle=-a_{\mathscr{Y}}\left(\widetilde{V}_{0}, W\right)+\left\langle f(0), \operatorname{tr}_{\Omega} W\right\rangle$ for all $W \in \mathbb{V}\left(\mathscr{T}_{Y}\right)$. Notice that $Z$ corresponds to a finite element approximation of $\operatorname{tr}_{\Omega} \partial_{t}^{2} \mathcal{U}(0)$.

If we consider $\widetilde{V}_{0}$ and $\widetilde{V}_{1}$, instead of $V_{0}$ and $V_{1}$, as initial data for the schemes of sections 6.4 and 6.5. then, to provide an a priori error analysis, it is necessary to modify the first two elements of the sequence $\Theta_{\Delta t}$, defined in (6.25), as follows:

$$
\Theta_{0}=\widetilde{V}_{0}-G_{\mathscr{T}_{y}} \mathcal{U}\left(t_{0}\right), \quad \Theta_{1}=\widetilde{V}_{1}-G_{\mathscr{T}_{y}} \mathcal{U}\left(t_{1}\right)
$$

In particular, it suffices to estimate

$$
E_{1}\left(\Theta_{\Delta t}\right)^{\frac{1}{2}}=\left(\frac{1}{2}\left\|\operatorname{tr}_{\Omega} \mathfrak{d} \Theta_{1}\right\|_{L^{2}(\Omega)}^{2}+\frac{1}{2}\left\|\Theta_{1 / 2}\right\|_{\mathcal{C}}^{2}\right)^{\frac{1}{2}} .
$$

We present the following error estimates.

Lemma 6.14 (error estimates for $\Theta_{0}$ and $\left.\Theta_{1}\right)$. If $\left(V_{0}, V_{1}\right)$ and $\left(\widetilde{V}_{0}, \widetilde{V}_{1}\right)$ are defined by (6.60) and (6.61), respectively, then

$$
\left\|\Theta_{0}\right\|_{\mathcal{C}} \lesssim h_{\mathscr{T}}\|g\|_{\mathbb{H}^{1+s}(\Omega)},
$$

and

$$
\left\|\Theta_{1}\right\|_{\mathcal{C}} \lesssim h_{\mathscr{T}} \mathfrak{A}(f, g, h)+(\Delta t)^{2} \Xi(f, g, h),
$$

where $\mathfrak{A}(f, g, h)$ and $\Xi(f, g, h)$ are defined by (4.20) and (4.10), respectively, and the the hidden constants are independent of $g, h,\left(V_{0}, V_{1}\right),\left(\widetilde{V}_{0}, \widetilde{V}_{1}\right)$, and $h_{\mathscr{T}}$.

Proof. Since $V_{0}, \tilde{V}_{0} \in \mathbb{V}\left(\mathscr{T}_{Y}\right)$, we can invoke property (6.6) and conclude that

$$
\begin{aligned}
\left\|\widetilde{V}_{0}-V_{0}\right\|_{\mathcal{C}}^{2} & =a_{\mathscr{Y}}\left(\widetilde{V}_{0}-V_{0}, \widetilde{V}_{0}-G_{\mathscr{T}_{y}} \mathcal{H}_{\alpha} \Pi_{x^{\prime}} g\right)+a_{\mathscr{Y}}\left(\widetilde{V}_{0}-V_{0}, G_{\mathscr{T}_{\gamma}} \mathcal{H}_{\alpha}\left(\Pi_{x^{\prime}} g-g\right)\right) \\
& =a_{\mathscr{Y}}\left(\widetilde{V}_{0}-V_{0}, \widetilde{V}_{0}-\mathcal{H}_{\alpha} \Pi_{x^{\prime}} g\right)+a_{\mathscr{Y}}\left(\widetilde{V}_{0}-V_{0}, \mathcal{H}_{\alpha}\left(\Pi_{x^{\prime}} g-g\right)\right)=\mathrm{I}+\mathrm{II}
\end{aligned}
$$

To bound I, we notice that $\operatorname{tr}_{\Omega}\left(\widetilde{V}_{0}-\mathcal{H}_{\alpha} \Pi_{x^{\prime}} g\right)=0$. On the other hand, $V_{0}-\widetilde{V}_{0}$ satisfies $a_{\mathscr{Y}}\left(V_{0}-\widetilde{V}_{0}, W\right)=0 \quad \forall W \in \mathbb{V}\left(\mathscr{T}_{Y}\right): \operatorname{tr}_{\Omega} W=0, \quad \operatorname{tr}_{\Omega}\left(V_{0}-\widetilde{V}_{0}\right)=\operatorname{tr}_{\Omega} G_{\mathscr{T}_{y}} \mathcal{H}_{\alpha} g-\Pi_{x^{\prime}} g$. 
Consequently, $\mathrm{I}=0$. Now, since $\mathcal{H}_{\alpha}$ satisfies that $\left\|\mathcal{H}_{\alpha} w\right\|_{\mathcal{C}} \lesssim\|w\|_{\mathbb{H}^{s}(\Omega)}$ for all $w \in$ $\mathbb{H}^{s}(\Omega)$, we arrive at

$$
|\mathrm{II}| \lesssim\left\|\widetilde{V}_{0}-V_{0}\right\|_{\mathcal{C}}\left\|g-\Pi_{x^{\prime}} g\right\|_{\mathbb{H}^{s}(\Omega)} \lesssim h_{\mathscr{T}}\left\|V_{0}-V_{0}\right\|_{\mathcal{C}}\|g\|_{\mathbb{H}^{1+s}(\Omega)}
$$

Since I $=0$, the estimate for II yields (6.62).

We now control $\left\|\Theta_{1}\right\|_{\mathcal{C}}$. A basic application of the triangle inequality together with estimate (6.36) reveal that

$$
\begin{aligned}
\left\|\Theta_{1}\right\|_{\mathcal{C}} & \leq\left\|\widetilde{V}_{1}-V_{1}\right\|_{\mathcal{C}}+\left\|V_{1}-G_{\mathscr{T}_{y}} \mathcal{U}\left(t_{1}\right)\right\|_{\mathcal{C}} \\
& \lesssim\left\|\widetilde{V}_{1}-V_{1}\right\|_{\mathcal{C}}+(\Delta t)^{3}\left\|\partial_{t}^{3} \nabla \mathcal{U}\right\|_{L^{\infty}\left(0, T ; L^{2}\left(y^{\alpha}, \mathcal{C}_{\gamma}\right)\right)}
\end{aligned}
$$

It thus suffices to bound $\left\|\widetilde{V}_{1}-V_{1}\right\|_{\mathcal{C}}$. To accomplish this task, we first notice that a simple application of Taylor's Theorem yields

$$
\begin{aligned}
& \widetilde{V}_{1}= \mathcal{H}_{\alpha}^{\mathscr{T}} \Pi_{x^{\prime}}\left[\operatorname{tr}_{\Omega} \mathcal{U}(0)+\Delta t \operatorname{tr}_{\Omega} \partial_{t} \mathcal{U}(0)+\frac{(\Delta t)^{2}}{2} \operatorname{tr}_{\Omega} \partial_{t}^{2} \mathcal{U}(0)\right] \\
&+\frac{(\Delta t)^{2}}{2} \mathcal{H}_{\alpha}^{\mathscr{T}}\left(Z-\Pi_{x^{\prime}} \operatorname{tr}_{\Omega} \partial_{t}^{2} \mathcal{U}(0)\right)=\mathcal{H}_{\alpha}^{\mathscr{T}} \Pi_{x^{\prime}}\left[\operatorname{tr}_{\Omega} \mathcal{U}\left(t_{1}\right)-\frac{(\Delta t)^{3}}{6} \operatorname{tr}_{\Omega} \partial_{t}^{3} \mathcal{U}(\bar{\zeta})\right] \\
&+\frac{(\Delta t)^{2}}{2} \mathcal{H}_{\alpha}^{\mathscr{T}}\left(Z-\Pi_{x^{\prime}} \operatorname{tr}_{\Omega} \partial_{t}^{2} \mathcal{U}(0)\right),
\end{aligned}
$$

with $\bar{\zeta} \in\left(0, t_{1}\right)$. Similar arguments allow us to conclude that

$$
V_{1}=G_{\mathscr{T}_{y}}\left(\mathcal{U}\left(t_{1}\right)-\frac{(\Delta t)^{3}}{6} \partial_{t}^{3} \mathcal{U}(\underline{\zeta})\right)
$$

with $\underline{\zeta} \in\left(0, t_{1}\right)$. Consequently,

$$
\begin{aligned}
\left\|\widetilde{V}_{1}-V_{1}\right\|_{\mathcal{C}} \lesssim \| \mathcal{H}_{\alpha}^{\mathscr{T}} \Pi_{x^{\prime}} & \operatorname{tr}_{\Omega} \mathcal{U}\left(t_{1}\right)-G_{\mathscr{T}_{Y}} \mathcal{U}\left(t_{1}\right)\left\|_{\mathcal{C}}+(\Delta t)^{3}\right\| \partial_{t}^{3} \nabla \mathcal{U} \|_{L^{\infty}\left(0, T ; L^{2}\left(y^{\alpha}, \mathcal{C}\right)\right)} \\
& +(\Delta t)^{2}\left\|\mathcal{H}_{\alpha}^{\mathscr{T}}\left(Z-\Pi_{x^{\prime}} \operatorname{tr}_{\Omega} \partial_{t}^{2} \mathcal{U}(0)\right)\right\|_{\mathcal{C}}=\mathrm{I}+\mathrm{II}+\mathrm{III} .
\end{aligned}
$$

To estimate I we invoke the same arguments that lead to (6.62):

$$
\mathrm{I} \lesssim h_{\mathscr{T}}\left\|\operatorname{tr}_{\Omega} \mathcal{U}\left(t_{1}\right)\right\|_{\mathbb{H}^{1+s}(\Omega)} \lesssim h_{\mathscr{T}}\left\|\operatorname{tr}_{\Omega} \mathcal{U}\right\|_{L^{\infty}\left(0, T ; \mathbb{H}^{1+s}(\Omega)\right)} .
$$

A bound for the term III follows from stability results. The collection of these estimates yield (6.63) $\mathrm{C}$

LEMMA 6.15 (estimate for $E_{1}\left(\Theta_{\Delta t}\right)$ ). If we consider $\widetilde{V}_{0}$ and $\widetilde{V}_{1}$ as initial data for the schemes of sections 6.4 and 6.5, we then have that

$$
\left\|\operatorname{tr}_{\Omega} \mathfrak{d} \Theta_{1}\right\|_{L^{2}(\Omega)}=\frac{1}{\Delta t}\left\|\operatorname{tr}_{\Omega}\left(\Theta_{1}-\Theta_{0}\right)\right\|_{L^{2}(\Omega)} \lesssim h_{\mathscr{T}}^{1+s} \mathfrak{A}(f, g, h)+(\Delta t)^{2} \Xi(f, g, h),
$$

and

$$
\left\|\Theta_{1 / 2}\right\|_{\mathcal{C}} \lesssim\left\|\Theta_{0}\right\|_{\mathcal{C}}+\left\|\Theta_{1}\right\|_{\mathcal{C}} \lesssim h_{\mathscr{T}} \mathfrak{A}(f, g, h)+(\Delta t)^{2} \Xi(f, g, h),
$$

where $\mathfrak{A}(f, g, h)$ and $\Xi(f, g, h)$ are defined by (4.20) and (4.10), respectively, and the the hidden constants are independent of $g, h,\left(V_{0}, V_{1}\right),\left(\widetilde{V}_{0}, \widetilde{V}_{1}\right)$, and $h_{\mathscr{T}}$

Proof. The proof of (6.68) follows directly from the estimates (6.62) and (6.63). In what follows we derive (6.67). To accomplish this task and simplify notation, we define

$$
\mathfrak{D}(\mathcal{U}):=\mathcal{U}(0)+\Delta t \partial_{t} \mathcal{U}(0)+\frac{1}{2}(\Delta t)^{2} \partial_{t}^{2} \mathcal{U}(0)
$$


Now, notice that

$$
\begin{aligned}
& \quad\left\|\operatorname{tr}_{\Omega}\left(\Theta_{1}-\Theta_{0}\right)\right\|_{L^{2}(\Omega)}=\left\|\operatorname{tr}_{\Omega}\left(\widetilde{V}_{1}-G_{\mathscr{T}_{y}} \mathcal{U}\left(t_{1}\right)-\widetilde{V}_{0}+G_{\mathscr{T}_{y}} \mathcal{U}(0)\right)\right\|_{L^{2}(\Omega)} \\
& \leq\left\|\operatorname{tr}_{\Omega}\left(\widetilde{V}_{1}-\mathfrak{D}\left(G_{\mathscr{T}_{y}} \mathcal{U}\right)-\widetilde{V}_{0}+G_{\mathscr{T}_{y}} \mathcal{U}(0)\right)\right\|_{L^{2}(\Omega)}+\left\|\operatorname{tr}_{\Omega}\left(\mathfrak{D}\left(G_{\mathscr{T}_{y}} \mathcal{U}\right)-G_{\mathscr{T}_{y}} \mathcal{U}\left(t_{1}\right)\right)\right\|_{L^{2}(\Omega)} .
\end{aligned}
$$

The trace estimate (2.10), the stability property (6.7) and an application of Taylor's Theorem reveal that

$$
\begin{aligned}
\left\|\operatorname{tr}_{\Omega}\left(\mathfrak{D}\left(G_{\mathscr{T}_{y}} \mathcal{U}\right)-G_{\mathscr{T}_{y}} \mathcal{U}\left(t_{1}\right)\right)\right\|_{L^{2}(\Omega)} & \lesssim\left\|\mathfrak{D}\left(G_{\mathscr{T}_{y}} \mathcal{U}\right)-G_{\mathscr{T}_{y}} \mathcal{U}\left(t_{1}\right)\right\|_{\mathcal{C}} \lesssim\left\|\mathfrak{D}(\mathcal{U})-\mathcal{U}\left(t_{1}\right)\right\|_{\mathcal{C}} \\
& \lesssim(\Delta t)^{3}\left\|\partial_{t}^{3} \nabla \mathcal{U}(\cdot, z)\right\|_{L^{\infty}\left(0, T ; L^{2}\left(y^{\alpha}, \mathcal{C}\right)\right)} .
\end{aligned}
$$

We now use the definitions of $\widetilde{V}_{0}$ and $\widetilde{V}_{1}$ to arrive at

$$
\begin{aligned}
\left\|\operatorname{tr}_{\Omega}\left(\widetilde{V}_{1}-\mathfrak{D}\left(G_{\mathscr{T}_{y}} \mathcal{U}\right)-\widetilde{V}_{0}+G_{\mathscr{T}_{y}} \mathcal{U}(0)\right)\right\|_{L^{2}(\Omega)} \leq(\Delta t)\left\|\Pi_{x^{\prime}} h-\operatorname{tr}_{\Omega} G_{\mathscr{T}_{y}} \partial_{t} \mathcal{U}(0)\right\|_{L^{2}(\Omega)} \\
+(\Delta t)^{2}\left\|Z-\operatorname{tr}_{\Omega} G_{\mathscr{T}_{y}} \partial_{t}^{2} \mathcal{U}(0)\right\|_{L^{2}(\Omega)} . \quad(6.70)
\end{aligned}
$$

To bound $\left\|\Pi_{x^{\prime}} h-\operatorname{tr}_{\Omega} G_{\mathscr{T}_{y}} \partial_{t} \mathcal{U}(0)\right\|_{L^{2}(\Omega)}$ we proceed as follows:

$$
\begin{aligned}
& \left\|\Pi_{x^{\prime}} h-\operatorname{tr}_{\Omega} G \mathscr{T}_{y} \partial_{t} \mathcal{U}(0)\right\|_{L^{2}(\Omega)} \leq\left\|\Pi_{x^{\prime}} h-h\right\|_{L^{2}(\Omega)} \\
& \quad+\left\|\operatorname{tr}_{\Omega} \partial_{t} \mathcal{U}(0)-\operatorname{tr}_{\Omega} G_{\mathscr{T}_{Y}} \partial_{t} \mathcal{U}(0)\right\|_{L^{2}(\Omega)} \lesssim h_{\mathscr{T}}^{1+s}\left(\|h\|_{\mathbb{H}^{1+s}(\Omega)}+\mathfrak{A}(f, g, h)\right),
\end{aligned}
$$

where we have used (6.27) with $\ell=1$. Similar arguments allow us to control $\| Z-$ $\operatorname{tr}_{\Omega} G_{\mathscr{T}_{y}} \partial_{t}^{2} \mathcal{U}(0) \|_{L^{2}(\Omega)}$. This concludes the proof.

REMARK 6.16 (influence of computable data in error estimates). If $\left(\widetilde{V}_{0}, \widetilde{V}_{1}\right)$ are used as initial data for the trapezoidal multistep method of section 6.4 and the leapfrog scheme of section 6.5. then the error estimates of Theorems 6.8 and 6.13 hold with no modifications.

7. Numerical results and implementation. Let $\left\{\phi_{1}, \ldots, \phi_{\mathcal{M}}\right\}$ denote a basis of $S^{\boldsymbol{r}}\left((0, \mathcal{Y}), \mathcal{G}_{\sigma}^{M}\right)$ such that $\phi_{1}(0)=1$ and $\phi_{j}(0)=0$ for $j>1$. The corresponding mass and stiffness matrices are denoted by $B_{y}$ and $A_{y}$ :

$$
\left(B_{\mathscr{Y}}\right)_{i j}=\int_{0}^{\mathscr{Y}} y^{\alpha} \phi_{i}(y) \phi_{j}(y) d y, \quad\left(A_{\mathscr{Y}}\right)_{i j}=\int_{0}^{\mathscr{Y}} y^{\alpha} \phi_{i}^{\prime}(y) \phi_{j}^{\prime}(y) d y .
$$

We denote by $B_{\Omega}$ and $A_{\Omega}$ the standard mass and stiffness matrices corresponding to the finite element space $S_{0}^{1}(\Omega, \mathscr{T})$. In what follows, we describe the implementation of a discrete Dirichlet-to-Neumann map. Once this operation is available the timestepping methods that are proposed in this work can be implemented in a standard way; in the case of the implicit method some further steps may be needed in order to obtain an efficient algorithm.

7.1. Discrete Dirichlet-to-Neumann map. Given $U \in S_{0}^{1}(\Omega, \mathscr{T})$, we consider the problem: Find $V \in \mathbb{V}\left(\mathscr{T}_{Y}\right)$ and $\eta \in S_{0}^{1}(\Omega, \mathscr{T})$ such that

$$
\begin{aligned}
a_{\mathscr{Y}}(V, W) & =\left\langle\eta, \operatorname{tr}_{\Omega} W\right\rangle \quad \forall W \in \mathbb{V}\left(\mathscr{T}_{Y}\right), \\
\operatorname{tr}_{\Omega} V & =U .
\end{aligned}
$$

Let us denote by $\mathbf{U}, \mathbf{V}$, and $\boldsymbol{\eta}$ the coefficient vectors associated with the discrete functions $U, V$ and $\eta$. Note that the first $N$ components of $\mathbf{V}$ and $\mathbf{U}$ are equal. We 
denote the remaining components of $\mathbf{V}$ by $\widetilde{\mathbf{V}}=(\mathbf{V})_{i}, i=N+1, \ldots, N \mathcal{M}$; we recall that $N=\# \mathscr{T}$, the number of degrees of freedom of $\mathscr{T}$. With this notation at hand, the matrix system (7.1) takes the form

$$
\left(B_{y} \otimes A_{\Omega}+A_{y} \otimes B_{\Omega}\right)\left(\begin{array}{c}
\mathbf{U} \\
\widetilde{\mathbf{V}}
\end{array}\right)=\left(\begin{array}{c}
B_{\Omega} \boldsymbol{\eta} \\
\mathbf{0}
\end{array}\right) .
$$

We denote by $\widetilde{B}_{y}$ and $\widetilde{A}_{y}$ the matrices that are obtained by removing the first row and first column from $B_{y}$ and $A_{y}$, respectively. Let $\tilde{b}_{y}$ and $\tilde{a}_{y}$ denote the vectors containing the first components of the rows $i=2, \ldots, \mathcal{M}$ of the matrices $B_{y}$ and $A_{y}$.

The vector $\widetilde{\mathbf{V}}$ is the solution of

$$
\left(\widetilde{B}_{y} \otimes A_{\Omega}+\widetilde{A}_{y} \otimes B_{\Omega}\right) \widetilde{\mathbf{V}}=-\left(\tilde{b}_{y} \otimes A_{\Omega}+\tilde{a}_{\mathscr{y}} \otimes B_{\Omega}\right) \mathbf{U} .
$$

Once this system is solved we can obtain $\boldsymbol{\eta}$ by solving

$$
B_{\Omega} \boldsymbol{\eta}=\left(b A_{\Omega}+a B_{\Omega}\right) \mathbf{U}+\left(\tilde{b}_{y}^{T} \otimes A_{\Omega}+\tilde{a}_{y}^{T} \otimes B_{\Omega}\right) \tilde{\mathbf{V}}
$$

where $b=\left(B_{Y}\right)_{11}$ and $a=\left(A_{\mathscr{Y}}\right)_{11}$. We denote by $L_{h}^{s}$ the matrix that describes the linear map $u \mapsto \eta$ :

$$
L_{h}^{s} \mathbf{U}=B_{\Omega} \boldsymbol{\eta}
$$

The main computational cost when solving (7.1) is the resolution of (7.2). This can be done efficiently by diagonalizing the small matrices $\widetilde{B}_{y}$ and $\widetilde{A}_{y}$. In fact, since both matrices are symmetric and positive definite, we can find a matrix of generalized eigenvectors $X$ such that

$$
X^{T} \widetilde{B}_{y} X=\operatorname{diag}\left(\mu_{1}, \ldots, \mu_{\mathcal{M}}\right), \quad X^{T} \widetilde{A}_{Y} X=\operatorname{diag}(1, \ldots, 1),
$$

where $\mu_{j}>0$ denote the eigenvalues of the generalized eigenvalue problem $\widetilde{B}_{\gamma} X=$ $\widetilde{A}_{\mathcal{Y}} X \operatorname{diag}\left(\mu_{1}, \ldots, \mu_{\mathcal{M}}\right)$. The system (7.2) can thus be transformed to $\mathcal{M}$ independent linear systems

$$
\left(\mu_{j} A_{\Omega}+B_{\Omega}\right) \hat{\mathbf{V}}_{j}=\left(\left(X^{T} \tilde{b}_{Y}\right)_{j} A_{\Omega}+\left(X^{T} \tilde{a}_{\mathscr{Y}}\right)_{j} B_{\Omega}\right) \mathbf{U},
$$

where $\hat{\mathbf{V}}^{T}=\left(\hat{\mathbf{V}}_{1}^{T}, \ldots, \hat{\mathbf{V}}_{\mathcal{M}}^{T}\right)$

$$
\widetilde{\mathbf{V}}=(X \otimes I) \hat{\mathbf{V}}
$$

and $I \in \mathbb{R}^{N \times N}$ is the identity matrix.

7.2. Leapfrog time-stepping scheme. Using the previously defined operator $L_{h}^{s}$, the leapfrog scheme can be now written in the familiar form

$$
\frac{1}{\Delta t^{2}} B_{\Omega}\left(\mathbf{U}_{k+1}-2 \mathbf{U}_{k}+\mathbf{U}_{k-1}\right)+L_{h}^{s} \mathbf{U}_{k}=B_{\Omega} \mathbf{f}_{k},
$$

where $\mathbf{f}_{k}$ is the coefficient vector containing the $L^{2}$ projection of $f\left(t_{k}\right)$ onto the space $S_{0}^{1}(\Omega, \mathscr{T})$. The main cost is the application of $L_{h}^{s} \mathbf{U}_{k}$ in each step followed by the inversion of the mass matrix $B_{\Omega}$; the latter being usually cheap. 
7.3. Trapezodial time-stepping scheme. The matrix system can be written, in a familiar form, involving only functions in $S_{0}^{1}(\Omega, \mathscr{T})$ :

$$
\frac{1}{\Delta t^{2}} B_{\Omega}\left(\mathbf{U}_{k+1}-2 \mathbf{U}_{k}+\mathbf{U}_{k-1}\right)+\frac{1}{4} L_{h}^{s}\left(\mathbf{U}_{k+1}+2 \mathbf{U}_{k}+\mathbf{U}_{k-1}\right)=\mathfrak{c} B_{\Omega} \mathbf{f}_{k} .
$$

The difficulty now is that at each step we need to solve the system

$$
\left(B_{\Omega}+\frac{\Delta t^{2}}{4} L_{h}^{s}\right) \mathbf{U}_{k+1}=\tilde{\mathbf{f}}_{k},
$$

where $\tilde{\mathbf{f}}_{k}$ contains known terms. While we could solve this system iteratively, it is more efficient to unwrap again the operator $L_{h}^{s}$ to see that $\mathbf{U}_{k+1}$ satisfies the system

$$
\left(B_{y} \otimes A_{\Omega}+A_{y} \otimes B_{\Omega}\right)\left(\begin{array}{c}
\mathbf{U}_{k+1} \\
\widetilde{\mathbf{V}}_{k+1}
\end{array}\right)=\left(\begin{array}{c}
B_{\Omega} \boldsymbol{\eta}_{k+1} \\
0
\end{array}\right)
$$

and

$$
B_{\Omega} \mathbf{U}_{k+1}+\frac{\Delta t^{2}}{4} B_{\Omega} \boldsymbol{\eta}_{k+1}=\tilde{\mathbf{f}}_{k} .
$$

Denoting by $E_{1}=\operatorname{diag}(1,0, \ldots, 0)$, we can write this as a single system

$$
\left(\frac{\Delta t^{2}}{4} B_{\mathfrak{Y}} \otimes A_{\Omega}+\left(E_{1}+\frac{\Delta t^{2}}{4} A_{\mathscr{Y}}\right) \otimes B_{\Omega}\right)\left(\begin{array}{l}
\mathbf{U}_{k+1} \\
\widetilde{\mathbf{V}}_{k+1}
\end{array}\right)=\left(\begin{array}{c}
\tilde{\mathbf{f}}_{k} \\
0
\end{array}\right) .
$$

As the matrices $B_{y}$ and $E_{1}+\frac{\Delta t^{2}}{4} A_{y}$ are again symmetric and positive definite similar diagonalization procedure can be used to solve the system efficiently.

\subsection{Numerical results.}

7.4.1. A $1 \mathrm{D}$ example. Let us first perform a numerical example for $n=1$. Let $\Omega=(0,1)$ and consider the space-fractional wave equation

$$
\partial_{t}^{2} u+(-\Delta)^{s} u=f
$$

with $f\left(x^{\prime}, t\right)=\left(\pi^{2 s}-1\right) \sin (t) \sin \left(\pi x^{\prime}\right)$. The initial data are such that the exact solution is $u\left(x^{\prime}, t\right)=\sin (t) \sin \left(\pi x^{\prime}\right)$. The initial values for both time-stepping schemes can be taken as $U_{0}=0$ and $U_{1}=\Delta t \Pi_{x^{\prime}} \sin \left(\pi x^{\prime}\right)$. Note that the additional $O\left(\Delta t^{2}\right)$ term that is needed in the definition of $U_{1}$ is zero because $u^{\prime \prime}(0)=0$.

We set the final time as $T=\pi / 2$ and perform numerical experiments for the following choices of the parameter $s: s=1 / 4$ and $s=3 / 4$. For space discretization in $\Omega$, we consider a uniform mesh with meshwidth $h_{\mathscr{T}}$. For the trapezoidal scheme, and to obtain linear convergence, we set $\Delta t=\left(0.5 h_{\mathscr{T}}\right)^{1 / 2}$. For the leapfrog scheme, in order to ensure the stability of the scheme, we choose $\Delta t=\left(0.5 h_{\mathscr{T}}\right)^{\max (1 / 2, s)}$. Notice that, the aforementioned choices of the parameter $s$ would render the leapfrog scheme unstable as a solution technique for the standard wave equation.

In Figure 7.1 we show the experimental rate of convergence for

$$
\text { error }=\left\|U_{K}-u(T)\right\|_{\mathbb{H}^{s}(\Omega)} .
$$

We observe that, as expected, the error decays linearly with respect to $h_{\mathscr{T}}$. We also notice that the errors for both discretization schemes are almost identical.

REMARK 7.1 (properties of the leapfrog scheme). When compared with the trapezoidal scheme, the leapfrog scheme is easier to implement. However, it seems to lose one of the main advantages that it has for the resolution of the standard wave equation. As the diffusion operator is nonlocal the explicit nature of the scheme is no longer of such an importance. 

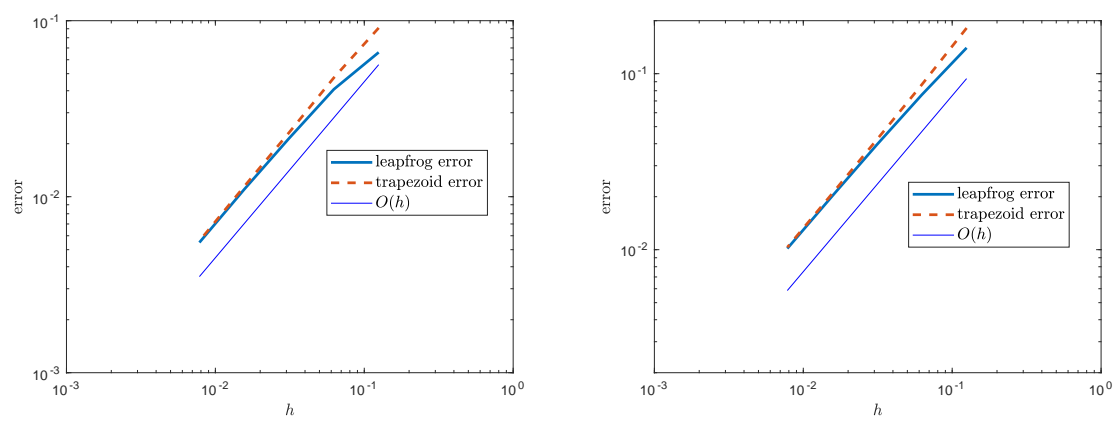

FIG. 7.1. Experimental rates of convergence for the error (7.4) for a one dimensional example. The experiment for $s=1 / 4$ is shown on the left and the one for $s=3 / 4$ on the right.

7.4.2. A $2 \mathrm{D}$ example. We let $n=2$, and consider the square domain $\Omega=$ $(-1,1)^{2} \subset \mathbb{R}^{2}$ and the space-fractional wave equation

$$
\partial_{t}^{2} u+(-\Delta)^{s} u=0
$$

The exact solution is given by

$$
u\left(x^{\prime}, t\right)=\cos \left(2^{s / 2} \pi^{s} t\right) \sin \left(\pi x_{1}^{\prime}\right) \sin \left(\pi x_{2}^{\prime}\right) .
$$

We set $T=1.5$ and $\Delta t=0.5 h_{\mathscr{T}}$. In this experiment we measure, in the $L^{2}(\Omega)$-norm, the error committed in the approximation of the time-derivative:

$$
\operatorname{error}_{2 D}=\left\|\mathfrak{d} U_{K}-\partial_{t} u\left(t_{K-1 / 2}\right)\right\|_{L^{2}(\Omega)} .
$$

The computations were done using the NGSolve/Netgen software package [46, 47].

The convergence properties of the leapfrog scheme are presented in Figure 7.2, It can be observed that, for both values of the parameter $s$ considered, the experimental rate of convergence for the $\operatorname{error}_{2 D}$ decays quadratically with respect to $h_{\mathscr{T}}$. We notice that the observed rates are better than the ones derived in Theorem 6.13 but are in agreement with approximation theory since, in this case, $u \in H^{2}(\Omega)$. The improvement of the estimate (6.58) of Theorem 6.13 from $h_{\mathscr{T}}^{1+s}$ to $h_{\mathscr{T}}^{2}$ is an open problem.

\section{REFERENCES}

[1] M. Abramowitz and I.A. Stegun. Handbook of mathematical functions with formulas, graphs, and mathematical tables. National Bureau of Standards Applied Mathematics Series. 1964.

[2] G. Acosta, F. Bersetche, and J.P. Borthagaray. Finite element approximations for fractional evolution problems. arXiv:1705.09815v1, 2017.

[3] I. Athanasopoulos and L.A. Caffarelli. Continuity of the temperature in boundary heat control problems. Adv. Math., 224(1):293-315, 2010.

[4] L. Banjai, J.M. Melenk, R.H. Nochetto, E. Otárola, A.J. Salgado, and Ch. Schwab. Tensor FEM for spectral fractional diffusion. arXiv:1707.07367v1, 2017.

[5] M.ŠS. Birman and M.Z. Solomjak. Spektralnaya teoriya samosopryazhennykh operatorov v gilbertovom prostranstve. Leningrad. Univ., Leningrad, 1980.

[6] M. Bonforte, Y. Sire, and J.L. Vázquez. Existence, uniqueness and asymptotic behaviour for fractional porous medium equations on bounded domains. Discrete Contin. Dyn. Syst., 35(12):5725-5767, 2015.

[7] X. Cabré and Y. Sire. Nonlinear equations for fractional Laplacians II: Existence, uniqueness, and qualitative properties of solutions. Trans. Amer. Math. Soc., 367(2):911-941, 2015. 


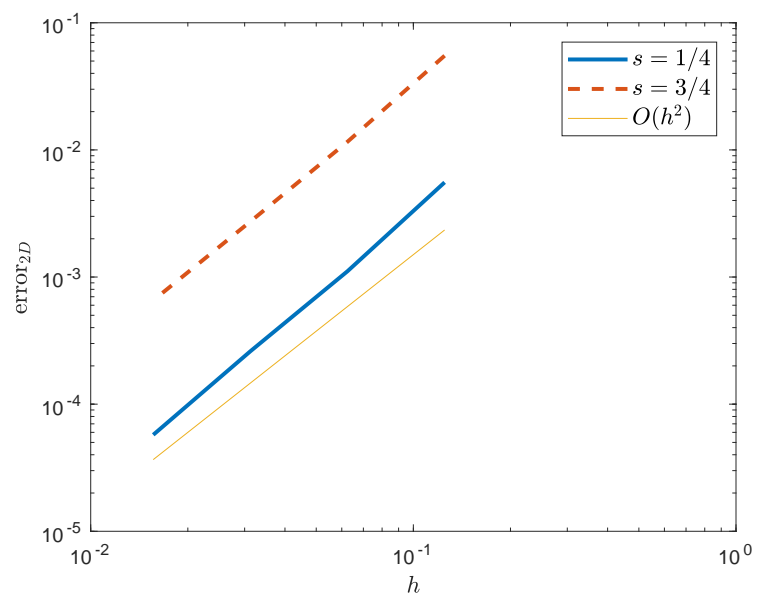

FIG. 7.2. Experimental rates of convergence for the error 17.5 when $\Omega=(-1,1)^{2}$ and for different values of $s$.

[8] X. Cabré and J. Tan. Positive solutions of nonlinear problems involving the square root of the Laplacian. Adv. Math., 224(5):2052-2093, 2010.

[9] L. Caffarelli and L. Silvestre. An extension problem related to the fractional Laplacian. Comm. Partial Differential Equations, 32(7-9):1245-1260, 2007.

[10] L. Caffarelli and P.R. Stinga. Fractional elliptic equations, Caccioppoli estimates and regularity. Ann. Inst. H. Poincaré Anal. Non Linéaire, 33(3):767-807, 2016.

[11] L. Caffarelli and A. Vasseur. Drift diffusion equations with fractional diffusion and the quasigeostrophic equation. Ann. of Math., 171:1903-1930, 2010.

[12] A. Capella, J. Dávila, L. Dupaigne, and Y. Sire. Regularity of radial extremal solutions for some non-local semilinear equations. Comm. Partial Differential Equations, 36(8):1353$1384,2011$.

[13] P. Carr, H. Geman, D.B. Madan, and M. Yor. The fine structure of asset returns: An empirical investigation. Journal of Business, 75:305-332, 2002.

[14] W. Chen. A speculative study of 2/3-order fractional Laplacian modeling of turbulence: Some thoughts and conjectures. Chaos, 16(2):1-11, 2006.

[15] W. Chen and S. Holm. Fractional laplacian time-space models for linear and nonlinear lossy media exhibiting arbitrary frequency power-law dependency. The Journal of the Acoustical Society of America, 115(4):1424-1430, 2004.

[16] W. Dahmen, B. Faermann, I. G. Graham, W. Hackbusch, and S. A. Sauter. Inverse inequalities on non-quasi-uniform meshes and application to the mortar element method. Math. Comp., 73(247):1107-1138, 2004.

[17] A. de Pablo, F. Quirós, A. Rodríguez, and J.L. Vázquez. A fractional porous medium equation. Adv. Math., 226(2):1378-1409, 2011.

[18] A. de Pablo, F. Quirós, A. Rodríguez, and J.L. Vázquez. A general fractional porous medium equation. Comm. Pure Appl. Math., 65(9):1242-1284, 2012.

[19] Q. Du, M. Gunzburger, R.B. Lehoucq, and K. Zhou. Analysis and approximation of nonlocal diffusion problems with volume constraints. SIAM Rev., 54(4):667-696, 2012.

[20] J. Duoandikoetxea. Fourier analysis, volume 29 of Graduate Studies in Mathematics. American Mathematical Society, Providence, RI, 2001. Translated and revised from the 1995 Spanish original by David Cruz-Uribe.

[21] A. Ern and J.-L. Guermond. Theory and practice of finite elements. Springer, New York, 2004.

[22] L.C. Evans. Partial differential equations, volume 19 of Graduate Studies in Mathematics. American Mathematical Society, Providence, RI, second edition, 2010.

[23] D. Fujiwara. Concrete characterization of the domains of fractional powers of some elliptic differential operators of the second order. Proc. Japan Acad., 43:82-86, 1967.

[24] P. Gatto and J.S. Hesthaven. Numerical approximation of the fractional laplacian via $h p$-finite elements, with an application to image denoising. J. Sci. Comput., 65(1):249-270, 2015.

[25] G. Gilboa and S. Osher. Nonlocal operators with applications to image processing. Multiscale 
Model. Simul., 7(3):1005-1028, 2008.

[26] V. Gol/dshtein and A. Ukhlov. Weighted Sobolev spaces and embedding theorems. Trans. Amer. Math. Soc., 361(7):3829-3850, 2009.

[27] P. Grisvard. Elliptic problems in nonsmooth domains, volume 69 of Classics in Applied Mathematics. Society for Industrial and Applied Mathematics (SIAM), Philadelphia, PA, 2011. Reprint of the 1985 original [ MR0775683], With a foreword by Susanne C. Brenner.

[28] G. Grubb. Fractional Laplacians on domains, a development of Hörmander's theory of $\mu$ transmission pseudodifferential operators. Adv. Math., 268:478 - 528, 2015.

[29] J. Heinonen, T. Kilpeläinen, and O. Martio. Nonlinear potential theory of degenerate elliptic equations. Oxford Mathematical Monographs. The Clarendon Press Oxford University Press, New York, 1993. Oxford Science Publications.

[30] T. Kato. Perturbation theory for linear operators. Classics in Mathematics. Springer-Verlag, Berlin, 1995. Reprint of the 1980 edition.

[31] A. Kiselev, F. Nazarov, and A. Volberg. Global well-posedness for the critical 2d dissipative quasi-geostrophic equation. Invent. Math., 167(3):445-453, Mar 2007.

[32] A. Kufner. Weighted Sobolev spaces, volume 31 of Teubner-Texte zur Mathematik [Teubner Texts in Mathematics]. BSB B. G. Teubner Verlagsgesellschaft, Leipzig, 1980. With German, French and Russian summaries.

[33] A. Kufner and B. Opic. How to define reasonably weighted Sobolev spaces. Comment. Math. Univ. Carolin., 25(3):537-554, 1984.

[34] N.S. Landkof. Foundations of modern potential theory. Springer-Verlag, New York-Heidelberg, 1972. Translated from the Russian by A. P. Doohovskoy, Die Grundlehren der mathematischen Wissenschaften, Band 180.

[35] S. Z. Levendorskiı̌. Pricing of the American put under Lévy processes. Int. J. Theor. Appl. Finance, 7(3):303-335, 2004.

[36] J.-L. Lions and E. Magenes. Non-homogeneous boundary value problems and applications. Vol. I. Springer-Verlag, New York-Heidelberg, 1972. Translated from the French by P. Kenneth, Die Grundlehren der mathematischen Wissenschaften, Band 181.

[37] W. McLean. Strongly elliptic systems and boundary integral equations. Cambridge University Press, Cambridge, 2000.

[38] B. Muckenhoupt. Weighted norm inequalities for the Hardy maximal function. Trans. Amer. Math. Soc., 165:207-226, 1972.

[39] R. Musina and A.I. Nazarov. On fractional Laplacians. Comm. Partial Differential Equations, 39(9):1780-1790, 2014.

[40] R. H. Nochetto, E. Otárola, and A. J. Salgado. A PDE approach to space-time fractional parabolic problems. SIAM J. Numer. Anal., 54(2):848-873, 2016.

[41] R.H. Nochetto, E. Otárola, and A.J. Salgado. A PDE approach to fractional diffusion in general domains: a priori error analysis. Found. Comput. Math., 15(3):733-791, 2015.

[42] F.W.J. Olver. Asymptotics and special functions. Academic Press [A subsidiary of Harcourt Brace Jovanovich, Publishers], New York-London, 1974. Computer Science and Applied Mathematics.

[43] E. Otárola and A.J. Salgado. Regularity of solutions to space-time fractional wave equations: a PDE approach. 2017. arXiv:1711.06186.

[44] H. Pham. Optimal stopping, free boundary, and American option in a jump-diffusion model. Appl. Math. Optim., 35(2):145-164, 1997.

[45] T. Roubíček. Nonlinear partial differential equations with applications, volume 153 of International Series of Numerical Mathematics. Birkhäuser/Springer Basel AG, Basel, second edition, 2013.

[46] J. Schöberl. NETGEN an advancing front 2D/3D-mesh generator based on abstract rules. $J$. Comput. Visual. Sci., 1:41-52, 1997.

[47] J. Schöberl. C ++11 implementation of finite elements in NGSolve. Technical report, 2014.

[48] S.A. Silling. Why peridynamics? In Handbook of peridynamic modeling, Adv. Appl. Math. CRC Press, 2017.

[49] P.R. Stinga and J.L. Torrea. Extension problem and Harnack's inequality for some fractional operators. Comm. Part. Diff. Eqs., 35(11):2092-2122, 2010.

[50] L. Tartar. An introduction to Sobolev spaces and interpolation spaces, volume 3 of Lecture Notes of the Unione Matematica Italiana. Springer, Berlin, 2007.

[51] B.O. Turesson. Nonlinear potential theory and weighted Sobolev spaces, volume 1736 of Lecture Notes in Mathematics. Springer-Verlag, Berlin, 2000.

[52] J.L. Vázquez and B. Volzone. Symmetrization for linear and nonlinear fractional parabolic equations of porous medium type. J. Math. Pures Appl. (9), 101(5):553-582, 2014. 